\title{
Spectral sequences in smooth generalized cohomology
}

\author{
DANIEL GRADY \\ HISHAM SATI
}

\begin{abstract}
We consider spectral sequences in smooth generalized cohomology theories, including differential generalized cohomology theories. The main differential spectral sequences will be of the Atiyah-Hirzebruch (AHSS) type, where we provide a filtration by the Čech resolution of smooth manifolds. This allows for systematic study of torsion in differential cohomology. We apply this in detail to smooth Deligne cohomology, differential topological complex K-theory, and to a smooth extension of integral Morava K-theory that we introduce. In each case we explicitly identify the differentials in the corresponding spectral sequences, which exhibit an interesting and systematic interplay between (refinement of) classical cohomology operations, operations involving differential forms, and operations on cohomology with $U(1)$ coefficients.
\end{abstract}

$55 \mathrm{~S} 25 ; 55 \mathrm{~N} 20,19 \mathrm{~L} 50$

\section{Contents}

1 Introduction 2

2 Smooth cohomology $\quad 8$

2.1 Smooth cohomology and stable category of smooth stacks _ . . . . 8

2.2 Differential cohomology and differential function spectra . . . . . 16

3 The smooth Atiyah-Hirzebruch spectral sequence (AHSS) 23

3.1 Construction of the spectral sequence via Čech resolutions . . . . . . 23

3.2 Morphisms of smooth spectral sequences and refinement of the AHSS 30

3.3 Product structure and the differentials $\ldots \ldots \ldots \ldots$ 
4 Applications to differential cohomology theories 37

4.1 Ordinary differential cohomology theory . . . . . . . . . . . 37

4.2 Differential $K$-theory . . . . . . . . . . . . . . . . . 41

4.3 Differential Morava K-theory . . . . . . . . . . . . . 51

$\begin{array}{ll}\text { Bibliography } & 57\end{array}$

\section{Introduction}

Spectral sequences are very useful algebraic tools that often allow for efficient computations that would otherwise require brute force (see [Mc01] for a broad survey). The Atiyah-Hirzebruch spectral sequence (henceforth AHSS) for K-theory and any generalized cohomology theory, in the topological sense, was introduced by Atiyah and Hirzebruch in [AH62a]. Excellent introduction to the generalized cohomology AHSS can also be found in Hilton [Hi71] and Adams [Ad74] (Sec. III.7). Other useful references on the subject include Switzer [Sw75] (Sec. 15, from a homology point of view, including the Gysin Sequence from AHSS) and interesting remarks in relation to spectra are given in Rudyak [Ru08] (Theorem 3.45 (homology), Remark 4.24 (Sheaves and Cech), Remark 4.34 (Postnikov), and Corollary 7.12). A description with an eye for applications is given in [HJJS08] (Ch. 21).

The goal of this paper is to systematically study the spectral sequence in the context of smooth or differential cohomology (see [CS85] [Fr00] [HS05] [SS08] [Bu12] [BS09] [Sc13]). Existence and interesting aspects of the AHSS in twisted forms of such differential cohomology theories have been considered briefly by Bunke and Nikolaus [BN14], where the main interest was the effect of the geometric part of the twist on the spectral sequence. In this paper we take a step back and consider untwisted differential generalized cohomology to systematically study the corresponding AHSS in generality and determine the differentials explicitly as cohomology operations. From the geometric point of view, one might expect on general grounds that the geometric information carried by the differential cohomology theory should somehow manifest itself within the spectral sequence. On the other hand, from an algebraic point of view, one might a priori not expect much of that information to be retained, or expect it to even be totally stripped out while running through the homological algebra machine. We will show that the answer lies somewhat in between, and both intuitions are to some extent correct: The differentials in the spectral sequence will be essentially 
refinements of classical ones, but with additional operations on differential forms. We recently characterized such operations in [GS16a], and so this paper is a natural continuation of that work.

Just as generalized cohomology theories are represented by spectra, differential cohomology theories are represented by certain sheaves of smooth spectra called differential function spectra. The original definition of differential function spectra was due to Hopkins and Singer in [HS05], generalized by Bunke, Nikolaus and Völkl in [BNV16] and was reformulated in terms of cohesion by Schreiber in [Sc13]. The terms smooth cohomology and differential cohomology seem to be used interchangeably in some of the literature (see e.g. [BS10]). However, we will find it useful for us to provide a specific and precise usage, where the first is viewed as being more general than the second. We also present most of our $\infty$-categories as combinatorial, simplicial model categories, rather than quasi-categories. We believe that this way nice objects are more easily and explicitly identifiable, which is something desirable when dealing with differential cohomology. Indeed, our discussion will be very explicit and the results will be readily utilizable.

Ordinary cohomology has smooth extension with various different realizations, including those of [CS85] [Ga97] [Br93] [DL05] [HS05] [BKS10]. All these realizations are in fact isomorphic [SS08] [BS10]. A description of K-theory with coefficients that combines vector bundles, connections, and differential forms into a topological context was initiated in [Ka87]. Using Karoubi's description Lott introduced $\mathbb{R} / \mathbb{Z}$-valued $\mathrm{K}$ theory [Lo94] as well as differential flat K-theory [Lo00]. Currently there are various geometric models of differential K-theory [Lo94] [BS09] [SS08] [FL10] [TWZ13] [TWZ15]. As in the case of ordinary differential cohomology these models should be equivalent. Indeed, explicit isomorphisms between various models have been demonstrated, for instance between the differential K-theory group of [HS05] and [FL10] in [K108], between Lott's $\mathbb{R} / \mathbb{Z}$ K-theory and Lott-Freed differential K-theory in the latter [FL10], the relation between Bunke-Schick differential K-theory and Lott(-Freed) differential K-theory is given in [Ho14], and the isomorphism between Simons-Sullivan [SS08] and Freed-Lott [FL10] is given in [Ho12].

The group structure of differential K-theory splits into odd and even degree parts, thus the refinement preserves the grading. However, the odd part turns out to be more delicate than the even part. In particular, while any two differential extensions of even $\mathrm{K}$-theory are isomorphic by the uniqueness results of [BS10], odd K-theory requires extra data in order to obtain uniqueness. There are various concrete models in the odd case, using smooth maps to the unitary group [TWZ13], via loop bundles [HMSV15], and via Hilbert bundles [GL15]. Our results in both even and odd K-theory will, of 
course, not depend on the particular model chosen.

Suppose $\mathcal{E}$ is a spectrum and $X$ is a space of the homotopy type of a CW-complex. Then there is a half-plane spectral sequence (AHSS)

$$
E_{2}^{p, q} \cong H^{p}\left(X ; \mathcal{E}^{q}(*)\right)
$$

converging conditionally to $\mathcal{E}^{*}(X)$. An immediate matter that we encounter in setting up the spectral sequence which calculates the generalized differential cohomology of a smooth manifold $X$ is how to deal with filtrations. Classically, Maunder [Ma63] gave two approaches to any generalized cohomology theory. The first is by filtering over the $q$-skeletons $X^{q}$ of the topological space $X$, and second by filtering over the Postnikov systems of spaces $Y_{q}$, which are the layers of an $\Omega$-spectrum associated to the cohomology theory. Maunder also gives an isomorphism between the two approaches. While we expect this to be the case in the differential setting, the proof might require considerable work. Hence, we leave this as an open problem. Maunder sets up his construction in the simplicial complex setting, which is equivalent to setting up in the CW-complex setting as the geometric realization of a simplicial set is a CWcomplex. Simplicial and Čech spectral sequences are discussed by May and Sigurdsson in [MS06] (Ch 22).

We will prefer the filtration of the spaces/manifolds rather than of the corresponding spectra, as this will naturally bring out the geometry desired in the smooth setting. We first would like to replace a topological space with skeletal filtration by a smooth manifold and then view this manifold as stack. Hence, in doing this, we need an analog of a skeleton in stacks. This will be done via Čech resolution of smooth spaces, and the replacement of skeletons of a space $X$ will be the various intersections of open sets covering the smooth manifold $X$.

We will use $\operatorname{diff}\left(\Sigma^{n} \mathcal{E}, \mathrm{ch}\right)$ to denote the differential refinement in degree $n$ of a cohomology theory $\mathcal{E}$. This was the notation used in [HS05] and carries more data than other notation, such as $\mathcal{E}(n)$. It also avoids possible confusion with other notations, e.g. when dealing with Morava K-theory $K(n)$ at chromatic level $n$. The axiomatic approach is very useful for characterizing a smooth cohomology theory, but one still needs the model of [HS05] for actually constructing examples of such smooth spectra. We will be using features of two main approaches at once, namely from [HS05] with $I: \operatorname{diff}\left(\Sigma^{n} \mathcal{E}, \mathrm{ch}\right) \rightarrow \underline{\mathcal{E}}$ and from [BNV16] [Sc13] with $I: \mathcal{E} \rightarrow \Pi \mathcal{E}$. Note that $\underline{\mathcal{E}}$ is not discrete while $\Pi \mathcal{E}$ is, but both are equivalent as smooth spectra $\underline{\mathcal{E}} \simeq \Pi \mathcal{E}$. This essentially boils down to the fact that since $\Pi \mathcal{E}$ is locally constant, the underlying theory satisfies $\Pi \mathcal{E}^{*}(U)=\Pi \mathcal{E}^{*}(*)$ on contractible open sets. On the other hand, the homotopy invariance of the theory $\underline{\mathcal{E}}$ implies the same thing: namely, $\underline{\mathcal{E}}(U) \simeq \underline{\mathcal{E}}(*)$, 
for a contractible $U$. These relationships are discussed in further detail in [BNV16].

We will be interested in how the differentials look like in our spectral sequences. One might a priori suspect that the differentials in the refined theories should at least loosely be connected to the differentials of the underlying topological theory. We will make this precise below, and so it seems appropriate to understand the form and structure of the differentials in the topological case. To illustrate the point, we will focus on what might perhaps be the most prominent example, namely the first differential $d_{3}: H^{*}\left(X, K^{0}(*)\right) \rightarrow H^{*}\left(X, K^{0}(*)\right)$ in complex topological K-theory $K(X)$ of a topological space $X$. This is given by $S q_{\mathbb{Z}}^{3}$ [AH62a] [AH62b]. There are exactly two stable cohomology operations $H^{*}(X ; \mathbb{Z}) \rightarrow H^{*+3}(X ; \mathbb{Z})$, since $H^{n+3}(K(\mathbb{Z}, n))=$ $\mathbb{Z} / 2$ for $n$ sufficiently large. One of these is zero and the other is $\beta \circ S q^{2} \circ \rho_{2}$, where $\beta$ is the Bockstein associated to the sequence $\mathbb{Z} \stackrel{\times 2}{\longrightarrow} \mathbb{Z} \stackrel{\rho_{2}}{\longrightarrow} \mathbb{Z}_{2}$ with $\rho_{2}$ denoting both the mod 2 reduction and its effect on cohomology with these as coefficients, i.e. $\rho_{2}: H^{i}(X ; \mathbb{Z}) \rightarrow H^{i}(X ; \mathbb{Z} / 2)$.

The above class, which is a priori in mod 2 cohomology, turned out to be a class in integral cohomology. One could work at any prime [AH62b], by noting the following (see e.g. [FFG86] or [Ha02]). For any class $x \in H^{n}(X ; \mathbb{Z} / p)$, and with $\beta_{p}$ the Bockstein associated with the sequence $\mathbb{Z}_{p} \stackrel{\times p}{\rightarrow} \mathbb{Z}_{p^{2}} \stackrel{\rho_{p}}{\rightarrow} \mathbb{Z}_{p}$, the elements $\beta_{p}(x)$ is an integral class in $H^{n+1}(X ; \mathbb{Z} / p)$, i.e. it belongs to the image of the reduction homomorphism $\rho_{p}: H^{n+1}(X ; \mathbb{Z}) \rightarrow H^{n+1}(X ; \mathbb{Z} / p)$. This can be used to prove the integrality of the class $d \in H^{3}(K(\mathbb{Z} / p, 2) ; \mathbb{Z} / p)$ as follows (see [FFG86]). The cohomology Serre spectral sequence for the path-loop fibration $\Omega K(\mathbb{Z}, 2) \rightarrow P K(\mathbb{Z}, 3) \rightarrow K(\mathbb{Z}, 3)$ gives that $H^{*}(K(\mathbb{Z}, 3) ; \mathbb{Z} / p)$ has a single additive generator $\bar{d}$ in dimension $\leq 2 p$. Now we have a map $\beta: K(\mathbb{Z} / p, 2) \rightarrow K(\mathbb{Z}, 3)$ such that $\beta^{*}(\bar{d})=d \in H^{3}(K(\mathbb{Z} / p, 2) ; \mathbb{Z} / p)$, constructed via the Serre spectral sequence of the path-loop fibration $K(\mathbb{Z} / p, 1) \rightarrow$ $P K(\mathbb{Z} / p, 2) \rightarrow K(\mathbb{Z} / p, 2)$. The map $\beta$ induces a map of loop spaces which are also Serre fibrations

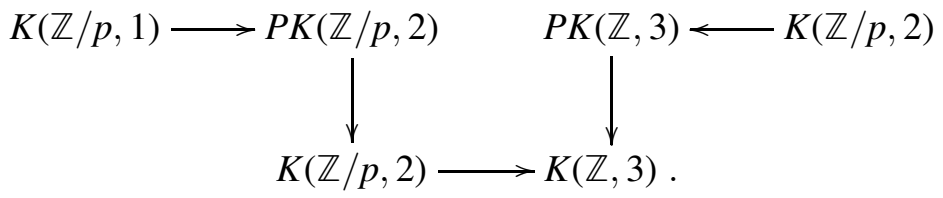

The induced homomorphism on the special sequences sends $\bar{d}$ to $d$ by the construction of $\beta$. Now we have $H^{3}(K(\mathbb{Z} / p, 2) ; \mathbb{Z} / p)=\mathbb{Z} / p$ hence $d$ is contained is contained in the image of the homomorphism $\rho_{p}: H^{3}(K(\mathbb{Z} / p, 2) ; \mathbb{Z}) \rightarrow H^{3}(K(\mathbb{Z} / p, 2) ; \mathbb{Z} / p)$. Therefore $d$ is an integral class. This is attractive as it makes it readily amenable to differential refinement. 
Such statements, and generalizations to other primes and to other generalized cohomology theories, can be made at the level of spectra (see e.g. [Sch]). The first nontrivial $k$-invariant of connective complex $\mathrm{K}$-theory spectrum $k u$ is a morphism $\mathbf{k}_{2}(k u) \in H^{2}(H \mathbb{Z}, \mathbb{Z})$, which is equal to $\beta \circ S q^{2}$, where $\beta: H \mathbb{Z} / 2 \rightarrow \Sigma(H \mathbb{Z})$ is the Bockstein operator associated to the extension $\mathbb{Z} \stackrel{\times 2}{\longrightarrow} \mathbb{Z} \longrightarrow \mathbb{Z} / 2$, and $S q_{\mathbb{Z}}^{2}$ is the pullback of the Steenrod operation $S q^{2} \in H^{2}(H \mathbb{Z} / 2, \mathbb{Z} / 2)$ along the projection morphism $\rho_{2}: H \mathbb{Z} \rightarrow H \mathbb{Z} / 2$ given by $\bmod 2$ reduction. Since $k u$ is a symmetric ring spectrum then, by [Sch] (Prop. 8.8), the $k$-invariants are derivations. The only derivations (up to units) in the $\bmod p$ Steenrod algebra $\mathcal{A}_{p}$ are the Milnor primitives $Q_{n} \in H^{2 p^{n}-1}(H \mathbb{Z} / p, \mathbb{Z} / p)$. At the lowest level we have $Q_{0}=\beta_{p}$ the $\bmod p$ Bockstein, and the others are realized as $k$-invariants of symmetric spectra, the connective Morava K-theory spectra $k(n)$. That is we have $Q_{n}=\mathbf{k}_{2 p^{n}-2}(k(n))$. We will consider refinements of integral lifts of these.

The classical AHSS collapses already at the first page if the generalized cohomology theory is rational. In fact, it can be shown that for any reasonably behaved spectrum like all the ones we consider, all the differentials in the AHSS are torsion, i.e. are zero when rationalized (see [Ru08] Cor. 7.12). The differentials in the AHSS in the topological case are analyzed by systematically by Arlettaz [Ar92]. Using the structure of the integral homology of the Eilenberg-MacLane spectra, it is proved there that for any connected space $X$ there are integers $R_{r}$ such that $R_{r} d_{r}^{s, t}$ for all $r \geq 2, s, t$. Some aspects of this general feature will continue to hold in the differential setting. Form a homotopy point of view there is not much difference between the localizations at $\mathbb{R}$ and at $\mathbb{Q}$. However, from a geometric point of view there is a considerable difference. Nevertheless, we will still use the term "rationalize" when we discuss localization at $\mathbb{R}$, as customary in the homotopy theory literature. We stress that the distinction is needed in certain geometric settings (see [GM81]), but it will not be an issue for us in this paper.

Note that although the differential cohomology diamond, i.e. the diagram that characterizes such theories (see Remark 6), certainly detects torsion classes in the flat part of the theory, it does not distinguish between torsion at various primes. As a by-product, our analysis can be seen as a systematic method for addressing $p$-primary torsion in differential theories. In [GS16a] we found that the Deligne-Beilinson squaring operation admits lower degree operations refining the Steenrod squares. We have the familiar pattern

$$
\mathrm{DD}, \widehat{S q}^{1}, \widehat{S q}^{2}, \widehat{S q}^{3}, \cdots \mathrm{DD}^{2}, \cdots
$$

where DD is the Dixmier-Douady class: a non-torsion differential cohomology oper- 
ation. The refined squares $\widehat{S q}^{2 k+1}$, as the classical squares $S q^{2 k}$, are operations that are 2-torsion. In this paper we get $\widehat{S q}^{2 k+1}$ as we expect, but also differentials $d_{2 m}$ at lowest degree for every $m$,

$$
d_{2 m}: \prod_{k} \Omega^{2 k}(M) \longrightarrow H^{2 m}(M ; U(1)) .
$$

We consider this as a cohomology operation, which can be viewed as first projecting on to the homogeneous component $\mathrm{ch}_{2 m}$ of the Chern-character. A $U(1)$-valued Čechcocycle is obtained by restricting to $2 m$-fold intersections of an open cover, pairing with an appropriate simplex of degree $2 m$ and exponentiating (This will be spelled out in detail in section 4). If indeed the form $\mathrm{ch}_{2 m}$ arises as the curvature of a bundle, it must represent a closed form with integral periods. The differential $d_{2 m}$ can therefore be understood as the obstruction to this condition. Similar results hold for the odd part, i.e. for differentially refined $K^{1}$-theory, where the refined Steenrod square takes the same form as in differential $K^{0}$-theory, while the differentials arising from forms the analogues of those in (1-1) - are now of odd degrees.

The paper is organized as follows. In Sec. 2 we start by carefully setting up the background in smooth and differential cohomology, preparing the scene for our constructions. In particular, in Sec. 2.1 we adapt abstract general results on stacks (or simplicial sheaves) to our context and spell out specific definitions and constructions that will be useful for us in later sections; more general and comprehensive accounts can be found in [Ja87] [Lu11] [Sc13]. Then in Sec. 2.2 we take the approach to differential cohomology that allows for a direct generalization. Our main constructions will be in Sec. 3, and in particular in Sec. 3.1 we provide the filtration via Čech resolutions and then construct the AHSS for smooth spectra in Sec. 3.2 and compare to the AHSS of the underlying topological theory. This refinement will depend on whether the degree is positive, negative, or zero. Then we explore the compatibility of the differentials with the product structure in Sec. 3.3.

Having given the main construction, our main applications of the general spectral sequence to various differential cohomology theories will be presented in Sec. 4. The construction is general enough to apply to any structured cohomology theory whose coefficients are known. We will explicitly emphasize three main examples: ordinary differential cohomology, differential K-theory, and a differential version of integral Morava K-theory that we introduce. As a test of our method, in Sec. 4.1 we recover the usual hypercohomology spectral sequence for the Deligne complex (see [Br93], [EV92] Appendix), and we do so for manifolds, then products of these, and then more generally for smooth fiber bundles. Then the AHSS for K-theory is generalized in Sec. 4.2 to 
differential K-theory, where the differential involve refinements of Steenrod squares, in the sense of [GS16a], as well as operations on forms, as indicated above around expression (1-1). We also show that the odd case, i.e. smooth extension of $K^{1}$, leads to a similar construction, but with the differentials now involving odd forms. Then in Sec. 4.3 we first introduce a refinement of the integral form of Morava K-theory, discussed in [KS03] [Sa10] [SW15], and then characterize the corresponding differentials, which turn out to have a similar pattern as in K-theory, where the operation that gets refined is the Milnor primitive $Q_{n}$ encountered above. We end with an application to an example from M-theory and string theory.

Notation. We have the following morphism that we will use repeatedly throughout. Denote by $\rho_{p}: \mathbb{Z} \rightarrow \mathbb{Z} / p$ the $\bmod p$ reduction on coefficients with corresponding morphism with the same notation on the cohomology groups with these as coefficients. We will denote by $\beta, \beta_{p}$, and $\tilde{\beta}$ the Bockstein homomorphisms associated with the coefficient sequences

$$
\begin{gathered}
0 \rightarrow \mathbb{Z} \rightarrow \mathbb{R} \stackrel{\exp }{\longrightarrow} U(1) \rightarrow 0, \\
0 \rightarrow \mathbb{Z} / p \stackrel{\times p}{\longrightarrow} \mathbb{Z} / p^{2} \stackrel{\rho_{p}}{\longrightarrow} \mathbb{Z} / p \rightarrow 0, \\
0 \rightarrow \mathbb{Z} \stackrel{\times p}{\longrightarrow} \mathbb{Z} \stackrel{\rho_{p}}{\longrightarrow} \mathbb{Z} / p \rightarrow 0,
\end{gathered}
$$

respectively. We will let $\Gamma_{2}: \mathbb{Z} / 2 \hookrightarrow U(1)$ denote the representation as the square roots of unity, also with $\Gamma_{2}: H^{n}(-; \mathbb{Z} / 2) \rightarrow H^{n}(-; U(1))$ the induced map on cohomology. We will also use more refined Bockstein homomorphisms associated with spectra, and these will be defined as we need them.

\section{Smooth cohomology}

\subsection{Smooth cohomology and stable category of smooth stacks}

In this section we adapt abstract general results on stacks (or simplicial sheaves) to our context and spell out specific definitions and constructions that will be useful for us in later sections. The interested reader can find more general and comprehensive accounts in [Ja87] [Lu11] [Sc13]. For the reader who is more interested in the applications to differential cohomology theories, this section can be skipped. However, we would like to emphasize that although the language used in this section is rather abstract, the generality gained from this formalism is far reaching and allows this machinery to be used for a wide variety theories, beyond just differential cohomology theories. 
Essentially, the axioms characterizing a smooth cohomology theory are not much different from the axioms characterizing usual cohomology theories. The big difference is where the theory takes place. More precisely, we want to consider homotopical functors on the category of pointed smooth stacks $S h_{\infty}(\text { CartSp })_{+}$with CartSp the category of Cartesian spaces, rather than the category of pointed topological spaces $\mathcal{T}_{\mathrm{op}}$. Let $\mathcal{A} \mathrm{b}_{g r}$ be the category of graded abelian groups.

Definition 1 (Smooth cohomology) Let $\mathcal{E}^{*}: S h_{\infty}(\operatorname{CartSp})_{+}^{\mathrm{op}} \rightarrow \mathcal{A} \mathrm{b}_{g r}$ be a functor satisfying the following axioms:

(1) (Invariance) $\mathcal{E}^{*}$ sends equivalences to isomorphisms.

(2) (Additivity) For small coproducts (i.e. ones forming sets) of pointed stacks, $\bigvee_{\alpha} X_{\alpha}$, we have

$$
\mathcal{E}^{*}\left(\bigvee_{\alpha} X_{\alpha}\right)=\prod_{\alpha} \mathcal{E}^{*}\left(X_{\alpha}\right)
$$

(3) (Mayer-Vietoris) For any homotopy pushout of pointed stacks,

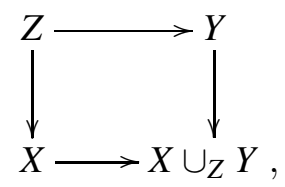

the induced sequence

$$
\mathcal{E}^{*}\left(X \cup_{Z} Y\right) \rightarrow \mathcal{E}^{*}(X) \oplus \mathcal{E}^{*}(Y) \rightarrow \mathcal{E}^{*}(Z)
$$

is exact.

(4) (Suspension) For any stack $X$, there is an isomorphism $\mathcal{E}^{n+1}(\Sigma X) \simeq \mathcal{E}^{n}(X)$.

Then we call $\mathcal{E}^{*}$ a smooth cohomology theory.

Remark 1 Note that the Mayer-Vietoris axiom implies the usual Mayer-Vietoris sequence. Indeed, let $M$ be a manifold and let $V$ be a local chart of $M$. Let $U$ be an open set such that $\{U, V\}$ is a cover of $M$. Then the strict pushout

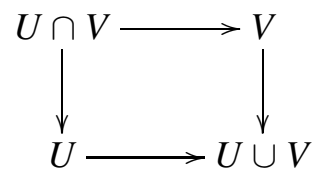

is actually a homotopy pushout. We can equivalently write this diagram as a homotopy coequalizer

$$
U \cap V \longrightarrow U \amalg V \longrightarrow U \cup V,
$$


in which the homotopy cofiber of the second map can be identified with $\Sigma U \cap V$. By iterating this argument and applying $\mathcal{E}^{*}$ to the the resulting diagram one obtains the long exact sequence

$$
\ldots \rightarrow \mathcal{E}^{*}(U \cap V) \rightarrow \mathcal{E}^{*}(M) \rightarrow \mathcal{E}^{*}(U) \oplus \mathcal{E}^{*}(V) \rightarrow \mathcal{E}^{*+1}(U \cap V) \rightarrow \ldots,
$$

which is the familiar Mayer-Vietoris sequence.

The above axioms can be taken as a generalization of the Eilenberg-Steenrod axioms (see [Ad74] [Hi71]), where the Mayer-Vietoris axiom subsumes both the excision axiom and the long-exact sequence axiom. It is interesting to note that the axioms $d o$ not require homotopy invariance. Namely, if two manifolds $M$ and $N$ are homotopic, they may fail to be equivalent as stacks. In fact, an equivalence of stacks requires, in particular, that for every sheaf $F$ (embedded as a stack), we have an isomorphism

$$
F(N) \simeq \pi_{0} \operatorname{Map}(N, F) \simeq \pi_{0} \operatorname{Map}(M, F) \simeq F(M) .
$$

In particular, we can take the sheaf of smooth $\mathbb{R}$-valued functions on a manifold. Then if every homotopy equivalence $f: M \rightarrow N$ induced an equivalence of stacks, we would have an induced isomorphism

$$
f^{*}: C^{\infty}(N ; \mathbb{R}) \rightarrow C^{\infty}(M ; \mathbb{R}) .
$$

Taking $N=*$ and $M=\mathbb{R}^{n}$ immediately gives a contradiction. On the other hand, every equivalence of stacks does produce a weak homotopy equivalence of geometric realizations. To see this, simply note that the geometric realization functor

$$
\Pi: S h_{\infty}(\text { CartSp }) \rightarrow s \mathcal{S e t},
$$

being a Quillen functor, has a derived functor by Ken Brown's Lemma [Br73]. It therefore preserves weak equivalences between fibrant objects. But these objects are exactly those that satisfy descent, namely stacks, (e.g. manifolds) [Sc13] [Du01].

Remark 2 Given a smooth cohomology theory $\mathcal{E}^{*}$, we always get a presheaf of graded abelian groups on the site Cartsp by precomposing with the Yoneda embedding:

$$
\mathcal{E}^{*}: \operatorname{Cart} S \mathrm{p} \stackrel{Y}{\longrightarrow} S h(\operatorname{CartSp}) \stackrel{\mathrm{sk}_{0}}{\longrightarrow} S h_{\infty}(\text { CartSp }) \stackrel{+}{\longrightarrow} S h_{\infty}(\text { CartSp })_{+} \stackrel{\mathcal{E}^{*}}{\longrightarrow} \mathcal{A} \mathrm{b}_{\mathrm{gr}}
$$

where $\mathrm{sk}_{0}$ embeds a sheaf as a discrete simplicial sheaf. We will use this fact later in the construction of the spectral sequence in theorem 11.

Just as all cohomology theories are representable by $\Omega$-spectra, via Brown representability, all smooth cohomology theories are representable by smooth spectra. This 
follows from the version of Brown representability formulated by Jardine in [Ja87] applied to the stable homotopy category of smooth stacks. We will quickly review the basic properties of this category (see [Lu11] [Ja15]) to establish where our objects of interest live.

We first recall some operations on stacks that are counterparts to standard operations on topological spaces. Let $X$ and $Y$ be two pointed stacks.

(i) The wedge product $X \vee Y$ is defined via the pushout diagram

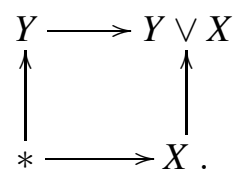

(ii) The smash product $X \wedge Y$ is defined as the quotient $X \wedge Y:=X \times Y / X \vee Y$ of the Cartesian product by the wedge product.

(iii) The suspension $\Sigma X$ is defined via the homotopy pushout diagram

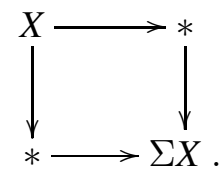

(iv) The looping, i.e. loop space, $\Omega X$ is defined via the homotopy pullback

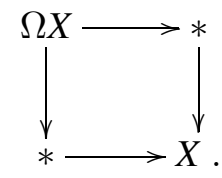

Definition 2 We define the stabilization $\mathrm{Stab}\left(S h_{\infty}(\operatorname{CartSp})_{+}\right)$of smooth pointed stacks to be the following category:

- The objects of $\operatorname{Stab}\left(S h_{\infty}(\text { CartSp })_{+}\right)$are sequences of pointed stacks

$$
\left\{\mathcal{E}_{n}\right\} \subset S h_{\infty}(\text { CartSp })_{+}, \quad n \in \mathbb{Z}
$$

equipped with maps $\sigma_{n}: \Sigma \mathcal{E}_{n} \rightarrow \mathcal{E}_{n+1}$.

- The morphisms between $\mathcal{E}$ and $\mathcal{F}$ are defined to to be the levelwise morphisms $\mathcal{E}_{n} \rightarrow \mathcal{F}_{n}$, commuting with the $\sigma_{n}$ 's.

This category carries a stable model structure given by first taking the projective model structure on sequences of stacks and then performing Bousfield localization with respect to stable weak equivalences in the usual way. This process is described in detail in 
[Ja87] [Lu11] [Ja15] and we summarize the relevant results found there. The category $\operatorname{Stab}\left(S h_{\infty}(\operatorname{CartSp})_{+}\right)$admits a stable, closed, simplicial model structure in which

- The weak equivalences are stable weak equivalences. That is, a morphism of smooth spectra $f: \mathcal{E}_{\bullet} \rightarrow \mathcal{F}_{\bullet}$ is a weak equivalence if and only if it induces a weak equivalence

$$
Q(f): \lim _{i \rightarrow \infty} \Omega^{i} \mathcal{E}_{n+i} \rightarrow \lim _{j \rightarrow \infty} \Omega^{j} \mathcal{F}_{n+j} .
$$

- The fibrant objects are precisely the smooth $\Omega$-Spectra, that is, the sequence of stacks $X$. whose structure maps

$$
\sigma_{n}: \Sigma \mathcal{E}_{n} \rightarrow \mathcal{E}_{n+1}
$$

induce equivalences $\mathcal{E}_{n} \stackrel{\sim}{\rightarrow} \Omega \mathcal{E}_{n+1}$.

Remark 3 We will refer to the stable model category $\operatorname{Stab}\left(S h_{\infty}(\text { CartSp })_{+}\right)$as the category of smooth spectra and denote it by

$$
S h_{\infty}(\operatorname{CartSp} ; \mathcal{S p}):=\operatorname{Stab}\left(S h_{\infty}(\operatorname{CartSp})_{+}\right) .
$$

Example 1 Let $M \in S h_{\infty}(\text { CartSp })_{+}$be a manifold, viewed a stack and equipped with a basepoint. We can define the smooth spectrum $\Sigma^{\infty} M$ in the usual way, as the sequence of suspensions of the manifold $M$. Given a smooth $\Omega$-spectrum $\mathcal{E}$, we can define a smooth cohomology theory $\mathcal{E}^{*}$, by setting

$$
\mathcal{E}^{q}(M) \simeq \pi_{0} \operatorname{Map}\left(\Sigma^{-q} \Sigma^{\infty} M, \mathcal{E}\right) .
$$

Differential cohomology theories are examples of the theories introduced above, although it may not be immediately apparent where the differential cohomology "diamond" diagram [SS08] fits into this context. In fact, it was observed by Bunke, Nikolaus and Völkl in [BNV16], that the diamond provides a further characterization of all smooth cohomology theories in terms of refinement of topological theories. This characterization happens in addition to the Brown representability described above, and happens only when the category of stacks exhibits so-called cohesion. We now review the properties of the cohesive structure on smooth stacks [Sc13] that we need, along with the characterization of smooth cohomology theories described in [BNV16]. It is shown in [Sc13] that the category $S h_{\infty}(\mathrm{Cart} S \mathrm{p})$ admits a quadruple $\infty$-categorical adjunction $(\Pi \dashv$ disc $\dashv \Gamma \dashv$ codisc $)$

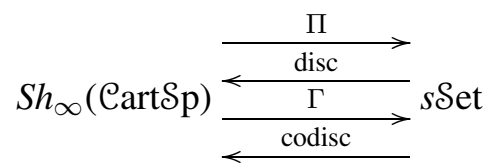


where $\Pi$ preserves finite $\infty$-limits and the functors disc and codisc are fully faithful.

One implication of this is that $s \mathcal{S}$ et embeds into $S h_{\infty}$ (CartSp) as an $\infty$-subcategory in two different ways, one reflective, the other reflective and coreflective. From the reflectors one can produce two monads and one comonad defined as follows:

$$
\Pi:=\Pi \circ \operatorname{disc}, \quad b:=\operatorname{disc} \circ \Gamma, \quad \sharp:=\operatorname{codisc} \circ \Gamma .
$$

These monads fit into a triple $\infty$-adjunction ( $\Pi \dashv b \dashv \sharp)$ which is called a cohesive adjunction.

Remark 4 Each monad in the cohesive adjunction picks out a different part of the nature of a smooth stack. This nature is perhaps best exemplified by how the adjoints behave on smooth manifolds (viewed as stacks). More precisely, if $M$ is a smooth manifold then, for instance,

(i) the comonad $b$ takes the underlying set of points of the manifold and then embedds this set back into stacks as a discrete object. This functor therefore misses the smooth structure of the manifold and treats it instead as a discrete object.

(ii) The monad $\Pi$ essentially takes the singular nerve of the maniflold using smooth paths and higher smooth simplices on the manifold. It therefore retains the geometry of the manifold and "knows" that the points of the manifold ought to be connected together in a smooth way.

The following observation on lifting from simplicial sets to spectra is known ([Sc13], Prop. 4.1.9), but we supply a proof for completeness.

Proposition 3 The $\infty$-adjunction (2-1) lifts to an $\infty$-adjunction

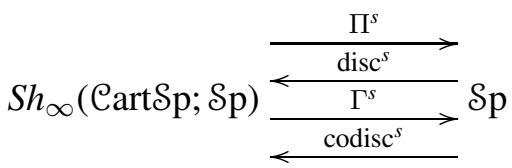

on the stable $\infty$-category of smooth spectra. Moreover, the adjoints satisfy the same condition as the $\infty$-adjunction (2-1) does.

Proof. The category of smooth stacks is presented by the combinatorial simplicial model category

$$
S h_{\infty}(\text { CartSp })=[\text { CartSp, } s \mathcal{S e t}]_{\text {loc,proj }}
$$

where loc denotes the Bousfield localized model structure at the maps out of Čech nerves. The quadruple adjunction is presented by Quillen adjoints $(\Pi \dashv$ disc $\dashv \Gamma \dashv$ 
codisc) [Sc13]. We need to show that this adjunction holds on the stable model category of smooth spectra. The adjunction immediately gives an underlying categorical adjunction by simply applying the functors degree-wise. In the projective model structure, the right adjoints are Quillen by definition and the closed model axioms imply that the left adjoints are also Quillen.

Now the functors (in the global model structure on $S p$ ) disc and codisc both preserve homotopy limits. Hence for a local weak equivalence $f: \mathcal{E} \rightarrow \mathcal{F}$ of spectra, we have

$$
\begin{aligned}
\lim _{i \rightarrow \infty} \Omega^{i} \operatorname{disc}(\mathcal{E})_{n+i} & \simeq \operatorname{disc}\left(\lim _{i \rightarrow \infty} \Omega^{i} \mathcal{F}_{n+i}\right) \\
& \simeq \operatorname{disc}\left(\lim _{j \rightarrow \infty} \Omega^{j} \mathcal{F}_{n+j}\right) \\
& \simeq \lim _{j \rightarrow \infty} \Omega^{j} \operatorname{disc}(\mathcal{F})_{n+j}
\end{aligned}
$$

and $\operatorname{disc}(f)$ induces a weak equivalence $Q(\operatorname{disc}(f))$. Hence, $\operatorname{disc}(f)$ is a weak equivalence. In the same way, codisc preserves local weak equivalences. It follows by the basic properties of Bousfield localization that disc and codisc are right Quillen adjoints. Again, by the axioms of a closed model category, it follows that the entire adjunction holds as Quillen adjunction of stable model categories.

Remark 5 The proof of the previous proposition implies that both disc and codisc preserve $\Omega$-spectra. However, $\Pi$ and $\Gamma$ need not take $\Omega$-spectra to $\Omega$-spectra. This problem can be remedied by taking $\Pi^{s}$ (or $\Gamma^{s}$ ) to be the composite of $R \circ \Pi$ (or $R \circ \Gamma$ ), where $R$ is the fibrant replacement in spectra. Since $R$ defines a left $\infty$-adjoint to the inclusion of fibrant objects (and preserves finite $\infty$-limits), we will still have an adjunction at the level of $\infty$-categories (although this is not presented by a Quillen adjunction).

As in the case of smooth stacks, the quadruple adjunction in the Proposition 3 produces adjoint monads $\left(\Pi^{s} \dashv b^{s} \dashv \sharp^{s}\right.$ ) exhibiting stable cohesion. The main observation in [BNV16], recast in the cohesive setting in [Sc13], is the following. Let $j: b^{s} \rightarrow$ id be the counit of the comonad $b^{s}$, and let $I:$ id $\rightarrow \Pi^{s}$ be the unit of the monad $\Pi^{s}$. Let 
$\mathcal{E} \in S h_{\infty}(\operatorname{Cart} S \mathrm{p} ; \mathcal{S p})$ be a smooth spectrum. Then $\mathcal{E}$ sits inside a hexagon diagram $(2-2)$

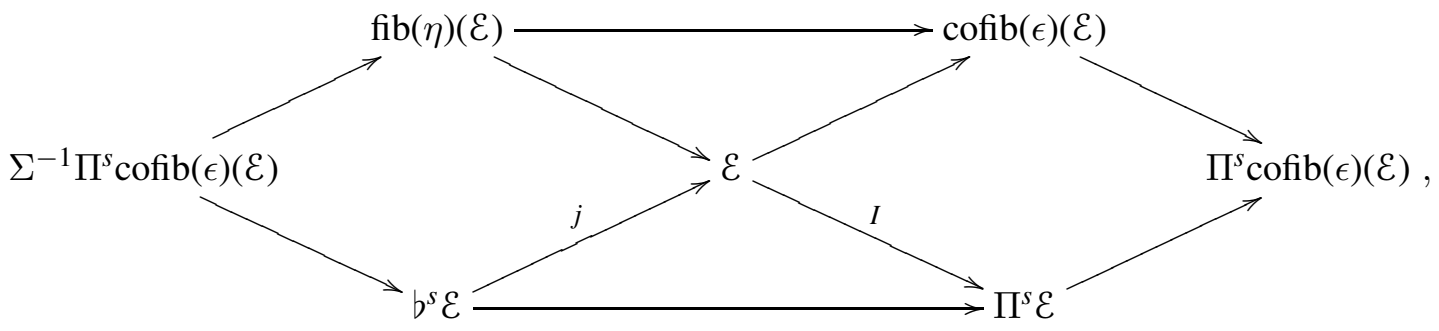

where the diagonals are fiber sequences (by definition), the top and bottom sequences are fiber sequences, and the two squares in the hexagon are homotopy Cartesian, i.e. both are homotopy pullback squares and hence homotopy pushout (via the equivalence of the two in the stable setting). The latter property is key, because it is a homotopy Cartesian square, as on the right of the hexagon, which Hopkins-Singer [HS05] took as the definition of differential cohomology (for a specific choice of the object of differential forms). Bunke-Nikolaus-Völkl [BNV16] observed that by the hexagon, every smooth spectrum satisfies this kind of Hopkins-Singer definition, if one just allows more general objects of differential forms, which is the object $\operatorname{cofib}(\epsilon)(\mathcal{E})$ in our notation above.

It often happens in practice that the smooth spectra $\operatorname{fib}(\eta)(\mathcal{E})$ and $\operatorname{cofib}(\epsilon)(\mathcal{E})$ contain no information away from degree 0 . In particular, it often happens that for $n>0$,

$$
\begin{aligned}
\pi_{n} \operatorname{Map}(M, \operatorname{cofib}(\epsilon)(\mathcal{E})) & \simeq 0, \\
\pi_{-n} \operatorname{Map}(M, \operatorname{fib}(\eta)(\mathcal{E})) & \simeq 0 .
\end{aligned}
$$

In this case the $\mathcal{E}$-cohomology of a manifold can be calculated as either the flat cohomology or the underlying topological cohomology in all degrees but 0 . This is summarized as the following result.

Proposition 4 Let $\mathcal{E}$ be a smooth spectrum such that (2-3) and (2-4) are satisfied. Then the $\mathcal{E}$-theory of a manifold $M$ is given by

$$
\mathcal{E}^{n}(M):= \begin{cases}\left(\Pi^{s} \mathcal{E}\right)^{n}(M) & n>0, \\ \left(b^{s} \mathcal{E}\right)^{n}(M) & n<0,\end{cases}
$$

(where in degree $0, \mathcal{E}(M)$ is already characterized by the diamond (2-2)).

Proof. Since the diagonals of the diamond are fiber sequences, they induce long exact 
sequences in cohomology. Let $n$ be a positive integer. The sequence

$$
b^{s} \mathcal{E} \rightarrow \mathcal{E} \rightarrow \operatorname{cofib}(\epsilon)(\mathcal{E})
$$

gives the section of the long sequence

$$
\pi_{n+1} \operatorname{Map}(M, \operatorname{cofib}(\epsilon)(\mathcal{E})) \rightarrow b^{s} \mathcal{E}^{-n}(M) \rightarrow \mathcal{E}^{-n}(M) \rightarrow \pi_{n} \operatorname{Map}(M, \operatorname{cofib}(\epsilon)(\mathcal{E})) .
$$

By assumption the leftmost and rightmost groups are 0 . We therefore have an isomorphism

$$
\left(b^{s} \mathcal{E}\right)^{-n}(M) \simeq \mathcal{E}^{-n}(M)
$$

Similarly, the sequence

$$
\mathrm{fib}(\eta)(\mathcal{E}) \rightarrow \mathcal{E} \rightarrow \Pi^{s} \mathcal{E}
$$

gives the long sequence

$$
\pi_{-n} \operatorname{Map}(M \operatorname{fib}(\eta)(\mathcal{E})) \rightarrow \mathcal{E}^{n}(M) \rightarrow\left(\Pi^{s} \mathcal{E}\right)^{n}(M) \rightarrow \pi_{-n-1} \operatorname{Map}(M, \operatorname{fib}(\eta)(\mathcal{E})),
$$

and again we get the desired isomorphism.

\subsection{Differential cohomology and differential function spectra}

The main applications we have in mind, as we indicated in the Introduction, concern differential cohomology theories. In this section we review some of the concepts established in [Bu12] [BNV16] [Sc13] (which generalize [SS08]), adapted to our context.

Definition 5 Let $\mathcal{E}^{*}$ be a cohomology theory. A differential refinement $\widehat{\mathcal{E}}^{*}$ of $\mathcal{E}^{*}$ consists of the following data:

(1) A functor $\widehat{\mathcal{E}}^{*}: S h_{\infty}\left(\operatorname{Cart} \mathrm{p}_{+}\right)^{\mathrm{op}} \rightarrow \mathcal{A} \mathrm{b}_{g r}$;

(2) Three natural transformations:

(a) Integration: $I: \widehat{\mathcal{E}}^{*} \rightarrow \mathcal{E}^{*}$;

(b) Curvature: $R: \widehat{\mathcal{E}}^{*} \rightarrow Z_{*}\left(\Omega^{*} \otimes \mathcal{E}^{*}(*)\right)$;

(c) Secondary Chern character: $a: \Omega^{*} \otimes \mathcal{E}^{*}(*)[1] / \operatorname{im}(d) \rightarrow \widehat{\mathcal{E}}^{*}$; such that the following axioms hold: 
- (Chern-Weil). We have a commutative diagram

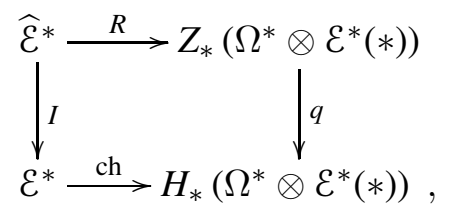

where ch is the Chern character map.

- (Secondary Chern-Weil). We have a commutative diagram

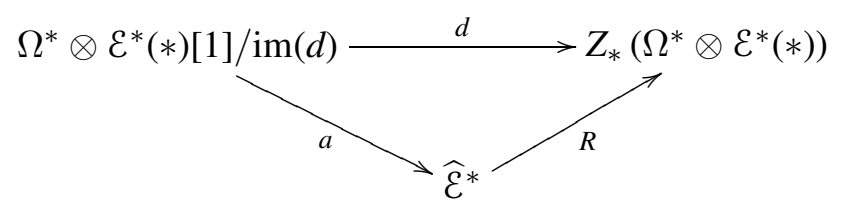

and an exact sequence

$$
\ldots \rightarrow \mathcal{E}^{*}[1] \rightarrow \Omega^{*} \otimes \mathcal{E}^{*}(*)[1] / \operatorname{im}(d) \rightarrow \widehat{\mathcal{E}}^{*} \rightarrow \mathcal{E}^{*} \rightarrow \ldots .
$$

Note that in item Chern-Weil above, $H_{*}\left(\Omega^{*} \otimes \mathcal{E}^{*}(*)\right)$ appears as the codomain of the Chern character. As explained in [BNV16], this becomes a locally constant stack equivalent to just the locally constant stack on the rationalization of $\mathcal{E}^{*}$, i.e., ch is equivalent to ch: $\mathcal{E}^{*} \rightarrow \mathcal{E}^{*} \wedge H \mathbb{R}$ (or $M \mathbb{R}$ ).

Remark 6 The above characterization can ultimately be summarized by saying that differential cohomology fits into an exact diamond

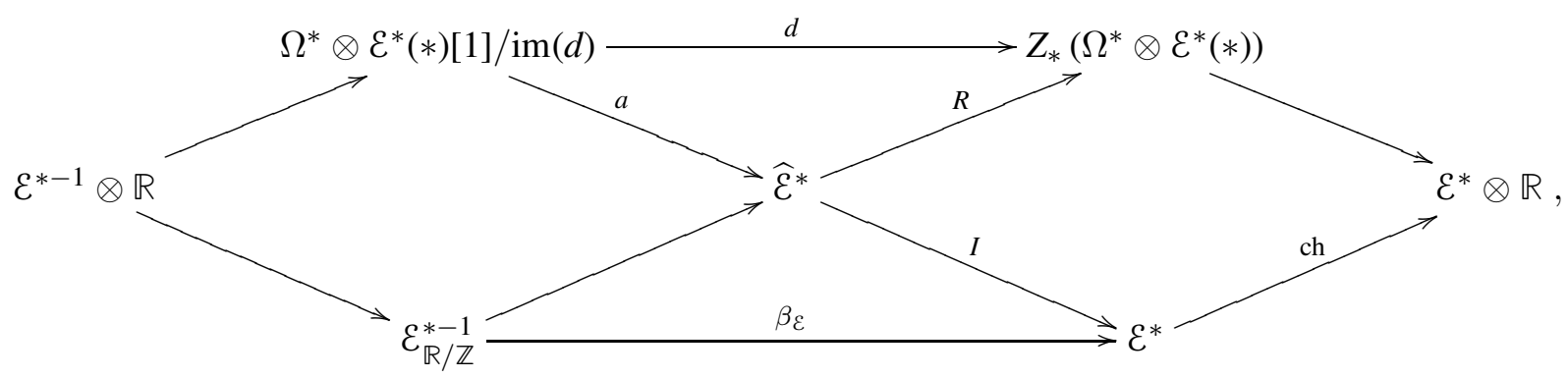

where the diagonal, top and bottom sequences are all part of long exact sequences. The bottom sequence is obtained by observing that the cofiber of the rationalization map is an $M U(1)$ (Eilenberg-Moore spectrum), where we identify $\mathbb{R} / \mathbb{Z}$ with $U(1)$ throughout. That is, we have a cofiber sequence involving the unit map from the sphere spectrum $\mathbb{S}=M \mathbb{Z}$

$$
\mathbb{S} \rightarrow M \mathbb{R} \rightarrow M U(1)
$$


Smashing on the left with the theory $\mathcal{E}$, we obtain a "Bockstein sequence"

$$
\mathcal{E} \rightarrow \mathcal{E} \wedge M \mathbb{R} \rightarrow \mathcal{E} \wedge M U(1) \stackrel{\beta_{\varepsilon}}{\longrightarrow} \Sigma \mathcal{E} .
$$

We define the flat theory as

$$
\mathcal{E}_{U(1)}:=\mathcal{E} \wedge M U(1)
$$

and the rational theory as

$$
\mathcal{E}_{\mathbb{R}}:=\mathcal{E} \wedge M \mathbb{R}
$$

Remark 7 Differential cohomology theories are a special case of smooth cohomology theories, while differential function spectra are a special case of smooth spectra. Thus, this section can be viewed as describing a special case of the previous section.

Since differential cohomology theories will arise as certain homotopy pullbacks (in Def. 7 below), we will first need to establish the components of the pullback. We begin with the following lemma that can be found in [Bu12] (Lemma 6.10), which explains how we can transition from a topological cohomology theory to a smooth one, in a process whose direction is opposite to that of the map $I$.

Lemma 6 Let $\mathcal{E}$ be a spectrum and define the smooth presheaf of spectra $\underline{\mathcal{E}}$ via the assignment

$$
\begin{aligned}
\text { Objects : } & U \mapsto \operatorname{Map}\left(\Sigma^{\infty} U, \mathcal{E}\right), \\
\text { Morphisms : } & (f: U \rightarrow V) \mapsto\left(f^{*}: \operatorname{Map}\left(\Sigma^{\infty} V, \mathcal{E}\right) \rightarrow \operatorname{Map}\left(\Sigma^{\infty} U, \mathcal{E}\right)\right) .
\end{aligned}
$$

Then $\underline{\mathcal{E}}$ satisfies descent.

Proof. Let $C^{\bullet}\left(\left\{U_{\alpha}\right\}\right)$ denote the Čech nerve of a good open cover $\left\{U_{\alpha}\right\}$ of some manifold $M$. The Yoneda Lemma and basic properties of the mapping space functor imply that we have the following sequence of equivalences

$$
\begin{aligned}
& \underline{\mathcal{E}}(M):=\operatorname{Map}\left(\Sigma^{\infty} M, \mathcal{E}\right) \\
& \simeq \operatorname{Map}\left(\Sigma^{\infty} \text { hocolim }_{\Delta^{o p}} C^{\bullet}\left(\left\{U_{\alpha}\right\}\right), \mathcal{E}\right) \\
& \simeq \operatorname{Map}\left(\operatorname{hocolim}_{\Delta^{o p}} \Sigma^{\infty} C^{\bullet}\left(\left\{U_{\alpha}\right\}\right), \mathcal{E}\right) \\
& \simeq \operatorname{holim}_{\triangle^{o p}} \operatorname{Map}\left(\Sigma^{\infty} C^{\bullet}\left(\left\{U_{\alpha}\right\}\right), \mathcal{E}\right) \\
& \simeq \operatorname{holim}\left\{\ldots \underset{\Longleftrightarrow}{\Longleftrightarrow} \prod_{\alpha \beta \gamma} \operatorname{Map}\left(\Sigma^{\infty} U_{\alpha \beta \gamma}, \mathcal{E}\right) \rightleftarrows \prod_{\alpha \beta} \operatorname{Map}\left(\Sigma^{\infty} U_{\alpha \beta}, \mathcal{E}\right) \rightleftarrows \prod_{\alpha} \operatorname{Map}\left(\Sigma^{\infty} U_{\alpha}, \mathcal{E}\right)\right\} \\
& \simeq \operatorname{holim}\left\{\ldots \underset{\sum}{\Longleftrightarrow} \prod_{\alpha \beta \gamma} \underline{\mathcal{E}}\left(U_{\alpha \beta \gamma}\right) \rightleftarrows \prod_{\alpha \beta} \underline{\mathcal{E}}\left(U_{\alpha \beta}\right) \rightleftarrows \prod_{\alpha} \underline{\mathcal{E}}\left(U_{\alpha}\right)\right\}
\end{aligned}
$$


and so $\underline{\mathcal{E}}$ satisfies descent.

The other components of the pullback we want to establish are presented by sheaves of chain complexes. There is a general functorial construction by which one can turn an unbounded chain complex into a spectrum, which we now describe (See [Sh07] for details). This functor is called the Eilenberg-MacLane functor

$$
H: \mathrm{Ch} \rightarrow \mathcal{S p}
$$

and acts on objects as follows. Let $C$. be an unbounded chain complex, and let $Z_{n}$ denote the subgroup of cycles in degree $n$. The functor $H$ takes $C$. and forms the sequence of truncated bounded chain complexes

$$
C \bullet \bullet \bullet=\left\{\begin{array}{l}
\left(\ldots \rightarrow C_{n} \rightarrow C_{n-1} \rightarrow \ldots C_{1} \rightarrow Z_{0}\right)=C_{\bullet}(0) \\
\left(\ldots \rightarrow C_{n} \rightarrow C_{n-1} \rightarrow \ldots C_{0} \rightarrow Z_{-1}\right)=C_{\bullet}(1) \\
\left(\ldots \rightarrow C_{n} \rightarrow C_{n-1} \rightarrow \ldots C_{-1} \rightarrow Z_{-2}\right)=C_{\bullet}(2) \\
\vdots \\
\left(\ldots \rightarrow C_{n} \rightarrow C_{n-1} \rightarrow \ldots C_{-k} \rightarrow Z_{-k}\right)=C \bullet(k) \\
\vdots
\end{array}\right.
$$

The reason for the group of cycles appearing in degree 0 comes from using the right adjoint to the inclusion $i: \mathrm{Ch}^{+} \rightarrow \mathrm{Ch}$ (as opposed to the left). The left adjoint simply truncates the complex in degree 0 , while the right adjoint truncates and then takes only the cycles in degree 0 .

Continuing with our discussion, at each level in the sequence, $H$ applies the Dold-Kan functor $D K: \mathrm{Ch}^{+} \rightarrow s \mathcal{S e t}$ to the bounded chain complex in that degree. This gives a sequence of spaces

$$
D K\left(C_{\bullet}(\bullet)\right)=\left\{\begin{array}{c}
D K(C \bullet(0)) \\
D K(C \bullet(1)) \\
D K\left(C_{\bullet}(2)\right) \\
\vdots \\
D K\left(C_{\bullet}(k)\right) \\
\vdots
\end{array}\right.
$$

Since $D K$ preserves looping (being a right Quillen adjoint) and equivalences (being a 
Quillen equivalence of model categories), we get induced equivalences

$$
\sigma_{k}: D K\left(C_{\bullet}(k)\right) \rightarrow \Omega D K(C \bullet(k-1)),
$$

which turns $D K(C \bullet(\bullet))$ into a spectrum.

Example 2 Consider the unbounded chain complex $\mathbb{Z}[0]$, with $\mathbb{Z}$ concentrated in degree 0 . Then

$$
H(\mathbb{Z}[0]) \simeq H \mathbb{Z}
$$

where the right hand side denotes the Eilenberg-MacLane spectrum.

Example 3 Fix a manifold $M$ and consider the de Rham complex

$$
\Omega^{*}:=\left(\ldots \rightarrow 0 \rightarrow 0 \rightarrow \Omega^{0}(M) \rightarrow \Omega^{1}(M) \rightarrow \ldots \Omega^{k}(M) \ldots\right),
$$

where the nonzero terms are concentrated in negative degrees. Then $H$ takes $\Omega^{*}$ to the spectrum

$$
H\left(\Omega^{*}(M)\right)=\left\{\begin{array}{l}
D K\left(\ldots \rightarrow 0 \rightarrow 0 \rightarrow \Omega_{\mathrm{cl}}^{0}(M)\right) \\
D K\left(\ldots \rightarrow 0 \rightarrow 0 \rightarrow \Omega^{0}(M) \rightarrow \Omega_{\mathrm{cl}}^{1}(M) \ldots\right) \\
D K\left(\ldots \rightarrow 0 \rightarrow 0 \rightarrow \Omega^{0}(M) \rightarrow \Omega^{1}(M) \rightarrow \Omega_{\mathrm{cl}}^{2}(M) \ldots\right) \\
\vdots \\
D K\left(\ldots \rightarrow 0 \rightarrow 0 \rightarrow \Omega^{0}(M) \rightarrow \Omega^{1}(M) \rightarrow \ldots \rightarrow \Omega_{\mathrm{cl}}^{k}(M) \ldots\right) \\
\vdots
\end{array}\right.
$$

By the basic properties of the Dold-Kan functor, the stable homotopy groups of this spectrum are computed as

$$
\begin{aligned}
\pi_{n}^{s} H\left(\Omega^{*}(M)\right) & \simeq \lim _{k \rightarrow \infty} \pi_{k+n} D K\left(\ldots \rightarrow 0 \rightarrow 0 \rightarrow \Omega^{0}(M) \rightarrow \Omega^{1}(M) \rightarrow \ldots \Omega_{\mathrm{cl}}^{k}(M)\right) \\
& \simeq \lim _{k \rightarrow \infty} H_{k+n}\left(\ldots \rightarrow 0 \rightarrow 0 \rightarrow \Omega^{0}(M) \rightarrow \Omega^{1}(M) \rightarrow \ldots \Omega_{\mathrm{cl}}^{k}(M)\right) .
\end{aligned}
$$

For $n>0$, these groups are 0 . For $n \leq 0$, they are the $n$th de Rham groups $H_{\mathrm{dR}}^{n}(M)$.

Now the functor $H$ in (2-5) prolongs to a functor on prestacks

$$
H:[\text { Cartsp, } \mathrm{Ch}] \rightarrow[\text { CartSp, Sp] . }
$$

In fact, using the properties of the Dold-Kan correspondence, it is fairly straightforward to show that this functor preserves local weak equivalences [Br73]. We therefore get a 
functor of smooth stacks

$$
H: S h_{\infty}(\text { CartSp; Ch }) \rightarrow S h_{\infty}(\text { CartSp; Sp }) .
$$

Recall that for an $\Omega$-spectrum $\mathcal{E}$, we always have a rational equivalence:

$$
\mathrm{r}: \mathcal{E} \wedge M \mathbb{R} \rightarrow H\left(\pi_{*}(\mathcal{E}) \otimes \mathbb{R}\right),
$$

where $M \mathbb{R}$ denotes an Eilenberg-Moore spectrum. Now, since we are working over the site of Cartesian spaces, the Poincaré lemma implies that the inclusion $j: \mathbb{R}[0] \rightarrow \Omega^{*}$ induces an equivalence

$$
\mathrm{id} \otimes j: \pi_{*}(\mathcal{E}) \otimes \mathbb{R}[0] \rightarrow \pi_{*}(\mathcal{E}) \otimes \Omega^{*},
$$

where $\pi_{*}(\mathcal{E})=\mathcal{E}(*)$ (which follows from suspension).

Definition 7 Let $\mathcal{E}$ be a spectrum. For an unbounded chain complex $C_{\bullet}$, let $\tau_{\leq 0} C_{\text {. }}$ denote the truncated complex

$$
\tau_{\leq 0} C_{\bullet}=\left(\ldots 0 \rightarrow 0 \rightarrow 0 \rightarrow C_{0} \rightarrow C_{-1} \rightarrow \ldots \rightarrow C_{-n} \rightarrow \ldots\right) .
$$

A differential function spectrum $\operatorname{diff}(\mathcal{E}, \mathrm{ch})$ is a homotopy pullback

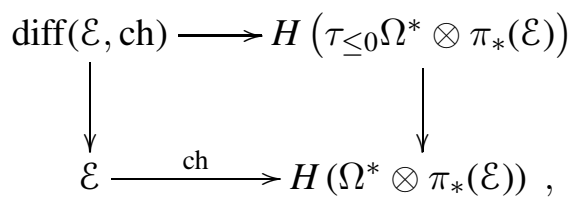

where $\mathrm{ch}=j \circ \mathrm{r}$ and $j$ induces an equivalence $j: \pi_{*}(\mathcal{E}) \otimes \mathbb{R}[0] \stackrel{\simeq}{\longrightarrow} \pi_{*}(\mathcal{E}) \otimes \Omega^{*}$.

Remark 8 In our definition, we have chosen the complex $\Omega^{*} \otimes \pi_{*}(\mathcal{E})$ as the de Rham complex modeling our rational theory. In general the differential function spectrum depends on this choice and on the equivalence $j$ [Bu12]. For the purposes of clarity and utility, we will always choose this model, although other models can be treated analogously. We do, however, keep the dependence on the map ch explicit to emphasize this fact.

Example 4 (Deligne cohomology) Let $\mathcal{E}=H(\mathbb{Z}[n]) \simeq \Sigma^{n} H \mathbb{Z}$ be the $n$-fold suspension of the Eilenberg-MacLane spectrum. In unbounded chain complexes, we have a natural isomorphism

$$
\underline{\mathbb{Z}}[n] \otimes \Omega^{*} \simeq \Omega^{*}[n],
$$

where $\mathbb{Z}[n]$ is the sheaf of locally constant integer-valued functions in degree $n$, and the complex on the right hand side has been shifted up $n$ units. That is $\Omega^{n}$ is in degree 
0 , while $\Omega^{0}$ is in degree $n$. Since $\Sigma^{n} H \mathbb{Z}$ is in the image of the Eilenberg-MacLane functor $H$ and $H$ preserves homotopy pullbacks, the homotopy pullback

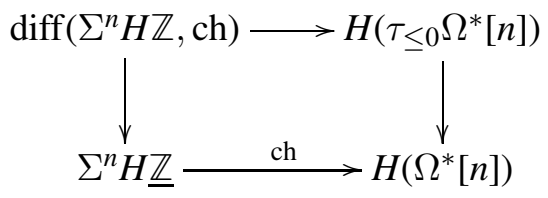

is presented by the homotopy pullback of unbounded chain complexes

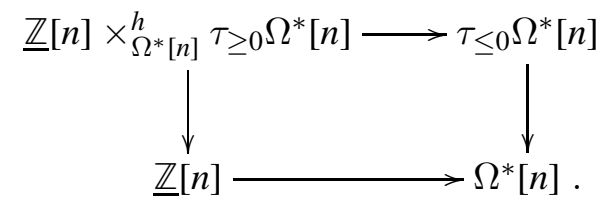

By stability, we can identify the homotopy pullback with the shifted mapping cone

$$
\underline{\mathbb{Z}}[n] \times_{\Omega^{*}[n]}^{h} \tau_{\leq 0} \Omega^{*}[n] \simeq \text { cone }\left(\underline{\mathbb{Z}}[n] \oplus \tau_{\leq 0} \Omega^{*} \rightarrow \Omega^{*}[n]\right)[-1] .
$$

The right hand side is precisely the Deligne complex $\mathbb{Z}_{\mathcal{D}}^{\infty}(n+1)$. We therefore have an equivalence

$$
H\left(\mathbb{Z}_{\mathcal{D}}^{\infty}(n+1)\right) \simeq \operatorname{diff}\left(\sum^{n} H \mathbb{Z}, \mathrm{ch}\right) .
$$

The underlying theory that this spectrum represents is precisely Deligne cohomology. In fact, by the Dold-Kan correspondence, we have an isomorphism of graded abelian groups

$$
\pi_{0} \operatorname{hom}_{\mathrm{Ch}}\left(N\left(C\left(\left\{U_{i}\right\}\right), \mathbb{Z}_{\mathcal{D}}^{\infty}(n+1)\right) \simeq \pi_{0} \operatorname{Map}\left(\Sigma^{\infty} M, \operatorname{diff}\left(\Sigma^{n} H \mathbb{Z}, \mathrm{ch}\right)\right) .\right.
$$

Here $N$ denotes the normalized Moore complex (adjoint to the Dold-Kan functor DK) and $C\left(\left\{U_{i}\right\}\right)$ denotes the $\breve{C}$ ech nerve of some good open cover of $X$. The right hand side is simply the definition of $\operatorname{diff}\left(\sum^{n} H \mathbb{Z}, \mathrm{ch}\right)^{0}(M)$, while the left hand side is the shifted total complex of the $\breve{C}$ ech Deligne double complex. It therefore computes the degree $n$ Deligne cohomology $H^{n}\left(M ; \mathbb{Z}_{\mathcal{D}}^{\infty}(n+1)\right)$.

The above example illustrates what exactly differential function spectra have to do with differential cohomology theories. The following definition can be found in [BNV16].

Definition 8 Let $\mathcal{E}$ be a spectrum and let

$$
\text { ch }: \mathcal{E} \rightarrow H\left(\tau_{\leq 0} \Omega^{*} \otimes \pi_{*}(\mathcal{E})\right),
$$

be the Chern character map as in Definition 7. The differential $\mathcal{E}$-cohomology of a 
manifold is the smooth cohomology theory with degree $n$ component

$$
\widehat{\mathcal{E}}^{n}(M) \simeq \operatorname{diff}\left(\Sigma^{n} \mathcal{E}, \mathrm{ch}\right)^{0}(M) .
$$

Since, for each $n, \operatorname{diff}\left(\sum^{n} \mathcal{E}, \mathrm{ch}\right)$ is a smooth spectrum it fits into a diamond diagram of the form (2-2), as established in [BNV16][Sch]. In [BNV16], it was shown that the form that this diamond takes is precisely the differential cohomology diamond in Remark 6. In particular, Proposition 4 allows us to calculate the $\operatorname{diff}\left(\sum^{n} \mathcal{E}, \mathrm{ch}\right)$ cohomology in degrees away from 0 as

$$
\operatorname{diff}\left(\Sigma^{n} \mathcal{E}, \mathrm{ch}\right)^{q}(M)=\left\{\begin{array}{cc}
\mathcal{E}^{n+q}(M) & q>0, \\
\mathcal{E}_{U(1)}^{n-1+q}(M) & q<0 .
\end{array}\right.
$$

\section{The smooth Atiyah-Hirzebruch spectral sequence (AHSS)}

In this section, we describe general machinery to construct an Atiyah-Hirzebruch spectral sequence (AHSS) from a smooth spectrum $\mathcal{E}$. We also describe how to compare this spectral sequence to the classical AHSS spectral sequence for the underlying theory $\Pi \mathcal{E}$, in nice cases.

\subsection{Construction of the spectral sequence via Čech resolutions}

The trick to describing the spectral sequence is to choose the right filtration on a fixed manifold. In the local (projective) model structure on smooth stacks, a natural choice arises: namely the Čech-type filtration on good open covers. This is indeed the most natural choice, since the maps which are weakly inverted in the local model structure are precisely those arising from taking the Čech nerve of a good open cover of a manifold. That is, we have a weak equivalence

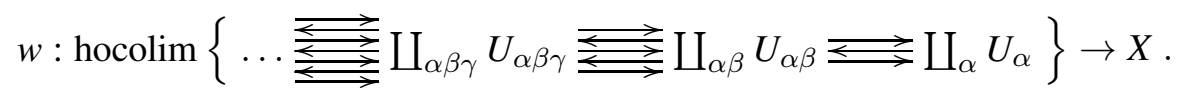

We now explicitly describe a filtration on $C\left(\left\{U_{i}\right\}\right)$. Recall that any simplicial diagram $J: \Delta^{\mathrm{op}} \rightarrow S h_{\infty}$ (CartSp) can be filtrated by skeleta. More precisely, let $i: \Delta_{\leq k} \hookrightarrow \Delta$ denote the embedding of the full subcategory of linearly ordered sets $[r]$, such that $r \leq k$. Then $i$ induces a restriction between functor categories (the $k$-th truncation)

$$
\tau_{\leq k}:\left[\Delta^{\mathrm{op}}, S h_{\infty}(\text { CartSp) }] \longrightarrow\left[\Delta_{\leq k}^{\mathrm{op}}, S h_{\infty}(\text { CartSp })\right] .\right.
$$


By general abstract nonsense (the existence of left and right Kan extensions), there are left and right adjoints $\left(\mathrm{sk}_{k} \dashv \tau_{\leq k} \dashv \operatorname{cosk}_{k}\right)$

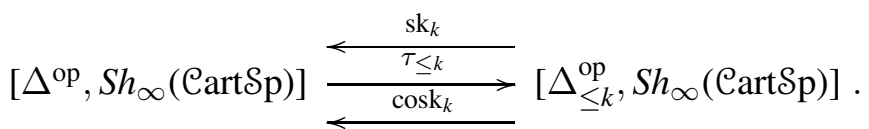

Furthermore, by composing adjoints, we have an adjunction $\left(\mathrm{sk}_{k} \dashv \operatorname{cosk}_{k}\right)$

$$
\left[\Delta^{\mathrm{op}}, S h_{\infty}(\text { Cartsp })\right] \underset{\mathrm{cosk}_{k}}{\stackrel{\mathrm{sk}_{k}}{\longleftarrow}}\left[\Delta^{\mathrm{op}}, S h_{\infty}(\operatorname{CartSp})\right] .
$$

The functor $\mathrm{sk}_{k}$ freely fills in degenerate simplices above level $k$, while $\operatorname{cosk}_{k}$ probes a simplicial object with simplices only up to level $k$ (the singular $k$-skeleton).

Proposition 9 Let $Y$. be a simplicial object in $S h_{\infty}$ (CartSp). Then we can filter $Y$. by skeleta

$$
\mathrm{sk}_{0} Y_{\bullet} \rightarrow \mathrm{sk}_{1} Y_{\bullet} \rightarrow \ldots \mathrm{sk}_{k} Y_{\bullet} \rightarrow \ldots \rightarrow Y_{\bullet} .
$$

The homotopy colimit over $Y_{\bullet}$ is presented by the ordinary colimit

$$
\underset{\Delta^{o p}}{\operatorname{hocolim}}\left(Y_{\bullet}\right) \simeq \underset{k \rightarrow \infty}{\operatorname{colim}} \underset{\Delta^{o p}}{\mathbb{c o l i m}}\left(\operatorname{sk}_{k} Y_{\bullet}\right),
$$

where $\llbracket$ colim is the left derived functor of the colimit, hence computable upon suitable cofibrant replacement of the diagram ${ }^{1}$.

Proof. Since $S h_{\infty}$ (CartSp) is presented by a combinatorial simplicial model category, the homotopy colimit over a filtered diagram is presented by the ordinary colimit and the canonical map

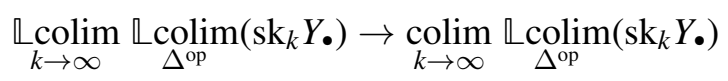

is an equivalence. Since homotopy colimits commute with homotopy colimits, we also have an equivalence

$$
\underset{k \rightarrow \infty}{\llbracket \operatorname{colim}} \underset{\Delta \text { op }}{\llbracket \operatorname{colim}}\left(\operatorname{sk}_{k} Y_{\bullet}\right) \simeq \underset{\Delta^{\text {op }}}{\mathbb{4} \operatorname{colim}} \underset{k \rightarrow \infty}{\llbracket \operatorname{colim}}\left(\operatorname{sk}_{k} Y_{\bullet}\right) .
$$

Again, using the fact that the ordinary colimit over a filtered diagram presents the homotopy colimit, we have an equivalence

$$
\underset{\Delta^{\text {op }}}{\mathbb{L} \operatorname{colim}} \underset{k \rightarrow \infty}{\mathbb{c} \operatorname{colim}}\left(\operatorname{sk}_{k} Y_{\bullet}\right) \rightarrow \underset{\Delta^{\text {op }}}{\mathbb{L} \operatorname{colim}} \underset{k \rightarrow \infty}{\operatorname{colim}}\left(\operatorname{sk}_{k} Y_{\bullet}\right) \simeq \underset{\Delta^{\text {op }}}{\mathbb{1} \operatorname{colim}}\left(Y_{\bullet}\right) .
$$

\footnotetext{
${ }^{1}$ We take this particular model of the homotopy colimit in order to ensure that taking the colimit of the resulting diagram makes sense. The claim will also hold for other presentations of the homotopy colimit
} 
Remark 9 The above proposition says that the homotopy colimit over the simplicial object is filtered by homotopy colimits of its skeleta. In particular, if $M$ is a paracompact manifold, we can fix a good open cover on $M$ and form the simplicial object given by its $\check{C}$ ech nerve

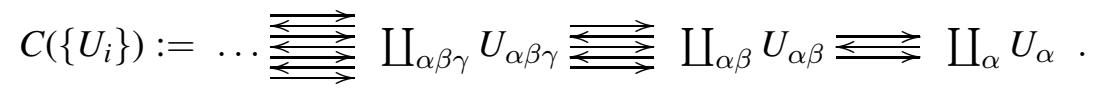

The homotopy colimit over this object is then filtered by its skeleta.

Let us see exactly what the skeleta look like in this case. To this end, we recall that in $S h_{\infty}$ (Cartsp) the full homotopy colimit is presented by the local homotopy formula

$$
\operatorname{hocolim}_{\Delta^{o p}} C\left(\left\{U_{i}\right\}\right)=\int^{n \in \Delta} \coprod_{\alpha_{0} \ldots \alpha_{n}} U_{\alpha_{0} \ldots \alpha_{n}} \odot \Delta[n] .
$$

The filtration on this object is given by first truncating the Čech nerve and then freely filling in degenerate simplices. As a consequence, in degree $k$ we can forget about the simplices of dimension higher than $k$. The homotopy colimit over this skeleton is then given by a strict colimit over the diagram

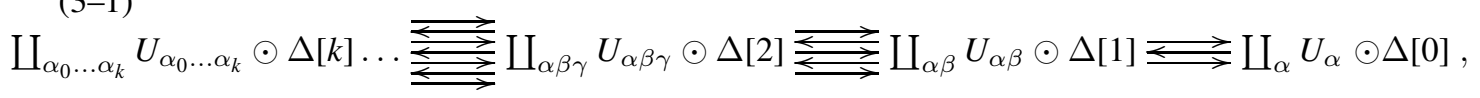

where the face and degeneracy maps are induced by the face and degeneracy maps of $\Delta[k]$. Taking $k \rightarrow \infty$, we do indeed reproduce the coend representing the full homotopy colimit $C\left(\left\{U_{i}\right\}\right)$.

We would like to eventually use this filtration to define a Mayer-Vietoris like spectral sequence for general cohomology theory $\mathcal{E}$. To get to this step, however, we will need to identify the successive quotients of the filtration. To simplify notation in what follows, we will fix a manifold $M$ with Čech nerve $C\left(\left\{U_{i}\right\}\right)$ and we set

$$
X_{k}:=\underset{\Delta^{o p}}{\operatorname{hocolim}}\left(\operatorname{sk}_{k} C\left(\left\{U_{i}\right\}\right)\right) .
$$

Then the quotient $X_{k} / X_{k-1}$ can be identified from the previous discussion by quotienting out the face maps at level $k$ described in diagram (3-1). Since the tensor of a simplicial set and a stack is given by the product of the stack with the discrete inclusion 
of the simplicial set, we can identify the quotient from the pushout of coends

$$
\begin{gathered}
\int^{n<k} \coprod_{\alpha_{0} \ldots \alpha_{n}} U_{\alpha_{0} \ldots \alpha_{n}} \times \operatorname{disc}(\Delta[n]) \longrightarrow * \\
\downarrow^{m \leq k} \underset{\alpha_{0} \ldots \alpha_{m}}{\coprod_{\alpha_{0} \ldots \alpha_{m}}} \times \operatorname{disc}(\Delta[m]),
\end{gathered}
$$

where $\partial$ denotes the boundary inclusion. At the level of points (or elements), a simplex in $\int^{n<k} \coprod_{\alpha_{0} \ldots \alpha_{k}} U_{\alpha_{0} \ldots \alpha_{n}} \times \operatorname{disc}(\Delta[n])$ is given by a pair

$$
(\rho, \sigma) \in \coprod_{\alpha_{0} \ldots \alpha_{k-1}} U_{\alpha_{0} \ldots \alpha_{k-1}} \times \operatorname{disc}(\Delta[k-1]),
$$

which is glued to lower simplices via the face and degeneracy relations.

Let us identify where the boundary inclusion takes a generic simplex. Then the quotient $X_{k} / X_{k-1}$ will be obtained by gluing these simplices together to a single point. Note that the face and degeneracy relations imply that simplices of the form $\left(\rho, s_{j+1} \sigma\right)$ are sent by $d_{j}$ to $\left(d_{j} \rho, \sigma\right)$. Since simplices in the image of the face maps are precisely those which are collapsed to a point, we see that

$$
\left(d_{j} \rho, \sigma\right) \sim * \text { for every } \sigma .
$$

We therefore see that each term of the coproduct $\coprod_{\alpha_{0} \ldots \alpha_{k}} U_{\alpha_{0} \ldots \alpha_{k}}$ is joined to another by the inclusion into a lower intersection. These lower intersections are then collapsed to a point yielding the wedge product

$$
\bigvee_{\alpha_{0} \ldots \alpha_{k}} U_{\alpha_{0} \ldots \alpha_{k}} \subset X_{k} / X_{k-1}
$$

Similarly, the simplex $\left(s_{j+1} \rho, \sigma\right)$ is sent to $\left(\rho, d_{j} \sigma\right)$ under $d_{j}$. We therefore identify the discrete simplicial sphere in the quotient

$$
\operatorname{disc}(\Delta[k] / \partial \Delta[k]) \subset X_{k} / X_{k-1} .
$$

Finally, the relations imposed by the coend imply that a simplex of the form $\left(s_{j} \rho, \sigma\right)$ is glued to $\left(\rho, d_{j} \sigma\right)$. The former are precisely those simplices in the simplicial sphere while the later are glued to the point. Similarly, $\left(\rho, s_{j} \sigma\right)$ is glued to the point. Thus we have the following.

Lemma 10 We can identify the quotient with the smash product

$$
X_{k} / X_{k-1} \simeq \operatorname{disc}(\Delta[k] / \partial \Delta[k]) \wedge \bigvee_{\alpha_{0} \ldots \alpha_{k}} U_{\alpha_{0} \ldots \alpha_{k}} \simeq \Sigma^{k}\left(\bigvee_{\alpha_{0} \ldots \alpha_{k}} U_{\alpha_{0} \ldots \alpha_{k}}\right) .
$$


Remark 10 (The filtration as a natural choice) Another way to think of our filtration above is the following. Let us form a Čech nerve of a manifold, then contract all the patches and intersections in that Čech nerve as points, such as to just obtain a simplicial set. Then the Borsuk's nerve theorem (see [Bj95] for a survey, [Ha02] Corollary 4G.3, or [Pr06] Theorem 3.21) says that this simplicial set is equivalent - weak homotopy equivalent - to the singular simplicial complex of the manifold, hence to its homotopy type. Moreover, that singular simplicial complex (or rather its geometric realization), in turn, gives a $\mathrm{CW}$-complex realization of the original manifold. So with this in mind, one may view our filtration above as the natural smooth refinement of the filteration by $C W$-stages of the manifold. That is, in taking the Čech nerve without contracting all its patches to points, we retain exactly the smooth information that, via Borsuk's theorem, corresponds to each cell in the canonical $\mathrm{CW}$-complex incarnation of the manifold. So in this sense, our refinement can be viewed as the canonical smooth refinement of the traditional filtering by $\mathrm{CW}$-stages.

We are now ready to describe the spectral sequence.

Theorem 11 (AHSS for general smooth spectra) Let $M$ be a compact smooth manifold and let $\mathcal{E}$ be a smooth spectrum. There is a spectral sequence with

$$
E_{2}^{p, q}=H^{p}\left(M, \mathcal{E}^{q}\right) \Longrightarrow \mathcal{E}^{p+q}(M) .
$$

Here $H^{p}$ denotes the $p$-th $\check{C}$ ech cohomology with coefficients in the presheaf $\mathcal{E}^{q}$. Moreover, the differential on the $E_{1}$-page is given by the differential in Čech cohomology.

Proof. The proof is almost immediate from the definitions. Recall that we have identified the quotients in Lemma 10. By the axioms for a smooth cohomology theory, we have that the $\mathcal{E}$-cohomology of the quotient is given by

$$
\begin{aligned}
\mathcal{E}^{*}\left(X_{k} / X_{k-1}\right) & \simeq \mathcal{E}^{*}\left(\Sigma^{k}\left(\bigvee_{\alpha_{0} \ldots \alpha_{k}} U_{\alpha_{0} \ldots \alpha_{k}}\right)\right) \\
& \simeq \mathcal{E}^{*-k}\left(\bigvee_{\alpha_{0} \ldots \alpha_{k}} U_{\alpha_{0} \ldots \alpha_{k}}\right) \\
& \simeq \bigoplus_{\alpha_{0} \ldots \alpha_{k}} \mathcal{E}^{*-k}\left(U_{\alpha_{0} \ldots \alpha_{k}}\right) .
\end{aligned}
$$

Applying $\mathcal{E}^{p+q}$ to the cofiber squence $X_{p} \hookrightarrow X_{p+1} \rightarrow X_{p+1} / X_{p}$ gives the long exact sequence in $\mathcal{E}$-cohomology

$$
\ldots \mathcal{E}^{p+q}\left(X_{p+1} / X_{p}\right) \rightarrow \mathcal{E}^{p+q}\left(X_{p+1}\right) \rightarrow \mathcal{E}^{p+q}\left(X_{p}\right) \rightarrow \mathcal{E}^{p+q+1}\left(X_{p+1} / X_{p}\right) \ldots
$$


Forming the corresponding exact triangle, we get a spectral sequence with $E_{1}^{p, q}$ term

$$
E_{1}^{p, q}=\bigoplus_{\alpha_{0}, \ldots, \alpha_{p}} \mathcal{E}^{q}\left(U_{\alpha_{0} \ldots \alpha_{p}}\right)
$$

Now we want to show that the differential on this page is given by the Čech differential

$$
\delta: E_{1}^{p, q}=\bigoplus_{\alpha_{0} \ldots \alpha_{p}} \mathcal{E}^{q}\left(U_{\alpha_{0} \ldots \alpha_{p}}\right) \longrightarrow \bigoplus_{\alpha_{0} \ldots \alpha_{p+1}} \mathcal{E}^{q}\left(U_{\alpha_{0} \ldots \alpha_{p+1}}\right)=E^{p+1, q} .
$$

To this end, note that differential on the $E_{1}$-page, by definition, comes from the exact sequence

$$
\ldots \rightarrow \mathcal{E}^{p+q}\left(X_{p+1} / X_{p}\right) \stackrel{j}{\rightarrow} \mathcal{E}^{p+q}\left(X_{p+1}\right) \stackrel{i}{\rightarrow} \mathcal{E}^{p+q}\left(X_{p}\right) \stackrel{\partial}{\rightarrow} \mathcal{E}^{p+q+1}\left(X_{p+1} / X_{p}\right) \rightarrow \ldots
$$

We need to show that $\partial j=d_{1}=\delta$ is the Čech differential. By naturality of the connecting homomorphism $\partial$, we have a commutative diagram

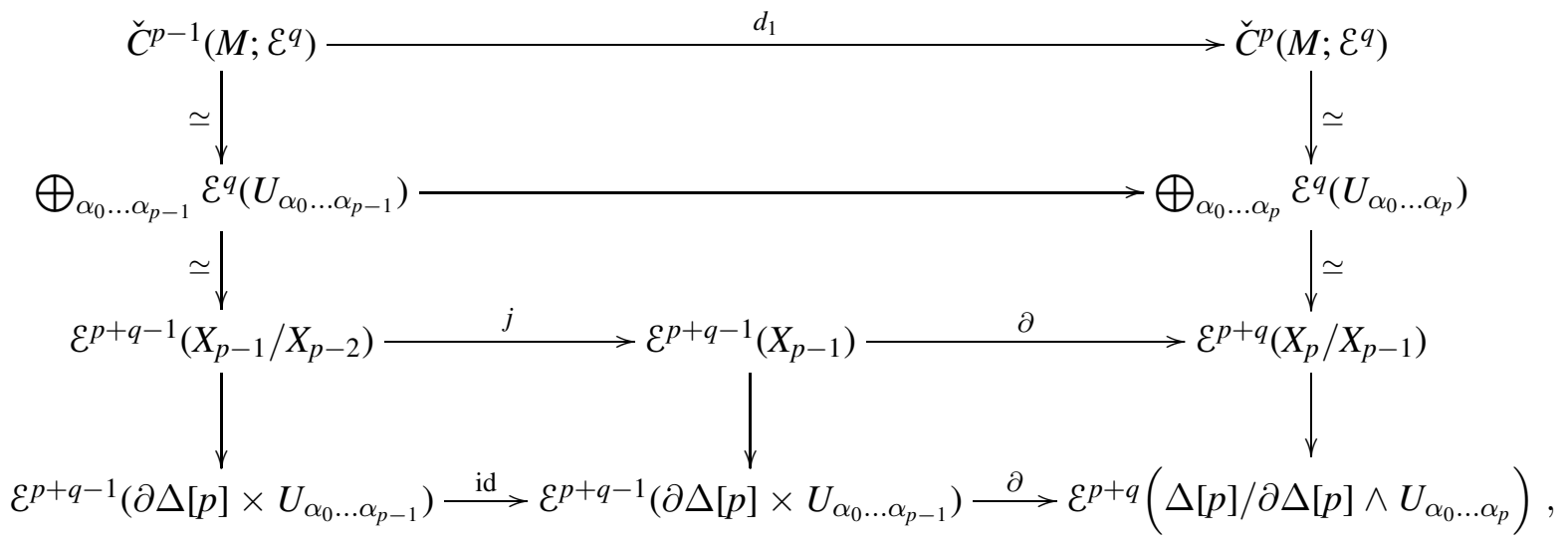

where the vertical bottom maps are induced from the inclusion of a factor

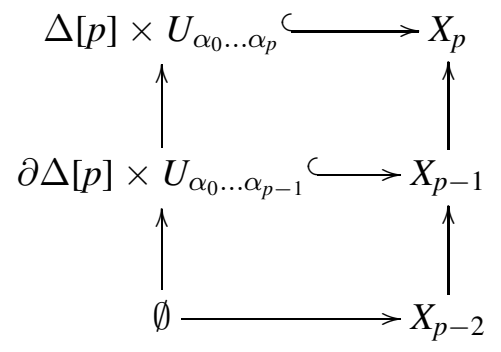

into the $p$-level of the filtration. Comparing the top and bottom composite morphisms in the big diagram, we see that on $(p-1)$-fold intersections $U_{\alpha_{0} \ldots \alpha_{p-1}}$, the map $d_{1}$ is forced to map a section to the alternating sum of restrictions, as this is precisely the 
map induced by the boundary inclusion in (3-3).

All that remains is the convergence. To establish that, we simply note that compactness implies that, for large values of $p$, we have an equivalence $X_{p} \simeq X$. Moreover, there are only finitely many diagonal entries at each page of the sequence. With this assumption, the convergence to the corresponding graded complex

$$
E_{\infty}^{p, q}=\frac{\operatorname{ker}\left(\mathcal{E}^{p+q}(X) \rightarrow \mathcal{E}^{p+q}\left(X_{p}\right)\right)}{\operatorname{ker}\left(\mathcal{E}^{p+q}(X) \rightarrow \mathcal{E}^{p+q}\left(X_{p+1}\right)\right)}=\frac{F_{p} \mathcal{E}^{p+q}(X)}{F_{p+1} \mathcal{E}^{p+q}(X)}
$$

follows exactly as in the classical case in [AH62a].

Fiber bundles. We can also construct a spectral sequence for a fiber bundle

$$
F \rightarrow N \stackrel{p}{\rightarrow} M
$$

where each map is a smooth map of manifolds and $M$ is compact. To that end, we note that for a fixed good open cover $\left\{U_{i}\right\}$ of $M$, the pullbacks $\left\{p^{-1}\left(U_{i}\right)\right\}$ define a good open cover of $N$. By local triviality, we have that each $p^{-1}\left(U_{i}\right) \simeq F \times U_{i}$. Then, using the filtration

$$
X_{k}=\underset{\Delta^{\mathrm{op}}}{\operatorname{hocolim}}\left(\operatorname{sk}_{k} C\left(\left\{p^{-1}\left(U_{i}\right)\right\}\right)\right)
$$

on the total space $N$, we identify the successive quotients

$$
X_{k} / X_{k-1} \simeq \Sigma^{k} \bigvee_{\alpha_{0} \ldots \alpha_{k}} U_{\alpha_{0} \ldots \alpha_{k}} \wedge F
$$

A similar argument as in the proof of Theorem 11 gives

Theorem 12 (Smooth AHSS for fiber bundles) Let $M, N$ and $F$ be manifolds, with $M$ compact. Let $F \rightarrow N \stackrel{p}{\rightarrow} M$ be a fiber bundle. Let $\mathcal{E}$ be a sheaf of spectra. Then there is a spectral sequence

$$
E_{2}^{p, q}=H^{p}\left(M, \mathcal{E}^{q}(-\wedge F)\right) \Longrightarrow \mathcal{E}^{p+q}(N) .
$$

Here $H^{p}$ denotes the $p$-th Čech cohomology with coefficients in the presheaf $\mathcal{E}^{-q}(-\wedge$ F).

Remark 11 (Unreduced theories) Note that the smooth spectral sequence works for reduced theories. One can treat unreduced theories similarly by setting

$$
\mathcal{E}^{q}(M, *):=\tilde{\mathcal{E}}^{q}\left(M_{+}\right),
$$


where the tilde denotes the reduced theory and $M_{+}$is the pointed stack with basepoint *. In this case, we have the slight modification on the second spectral sequence, which takes the form

$$
E_{2}^{p, q}=H^{p}\left(M, \mathcal{E}^{q}(-\times F)\right) \Longrightarrow \mathcal{E}^{p+q}(N)
$$

\subsection{Morphisms of smooth spectral sequences and refinement of the AHSS}

Our next task will be to show that these spectral sequences do indeed refine the classical Atiyah-Hirzebruch spectral sequence (AHSS) [AH62a]. Since any smooth theory $\mathcal{E}$ comes as a refinement of the underlying topological theory $\Pi \mathcal{E}$, we will immediately get a morphism of spectral sequences induced by the morphism of spectra

$$
I: \mathcal{E} \rightarrow \Pi \mathcal{E} .
$$

Unfortunately, this morphism does not allow us to compare the differentials of the spectral sequences in the way that we would ideally hope for. However, as we will progressively see, the situation can be remedied by constructing a slightly different morphism of spectral sequences. This morphism is related to the boundary map of spectral sequences which occurs when a morphism of spectra induces the 0 map on corresponding spectral sequences (see [Mi81] for a discussion in the case of the Adams spectral sequence). We first discuss the morphism induced by $I$ and then construct this "boundary type" map and prove that it indeed defines a morphism of spectral sequences.

Definition 13 Let $E_{n}^{p, q}$ and $F_{n}^{p, q}$ be spectral sequences, that is, a sequence of bigraded complexes $E_{n}^{p, q}$ and $F_{n}^{p, q}, n \in \mathbb{N}$. A morphism of spectral sequences is a morphism of bigraded complexes

$$
f_{n}: E_{n}^{p, q} \rightarrow F_{n}^{p, q},
$$

defined for all $n>N$, where $N$ is some fixed positive integer. Furthermore, we require the map $f_{n+1}$ to be the map on homology induced by $f_{n}$. We call the smallest integer $N$ such that $f_{n}$ are defined for $n>N$ the rank of the morphism.

We now apply this to the smooth AHSS. The next result should follow from general principles, but we emphasise it explicitly for clarity and for subsequent use.

Proposition 14 Let $\mathcal{E}$ and $\mathcal{F}$ be smooth spectra. Then a map $f: \mathcal{E} \rightarrow \mathcal{F}$ induces a morphism of corresponding smooth AHSS's

$$
E_{n}^{p, q} \rightarrow F_{n}^{p, q}
$$


Proof. Fix a manifold $X$ and a good open cover $\left\{U_{i}\right\}$. Let $X_{p}$ denote the $p$-th filtration of the Čech nerve as before. It is clear by naturality that a map of spectra $f: \mathcal{E} \rightarrow \mathcal{F}$ induces a morphism of long exact sequences (see (3-2))

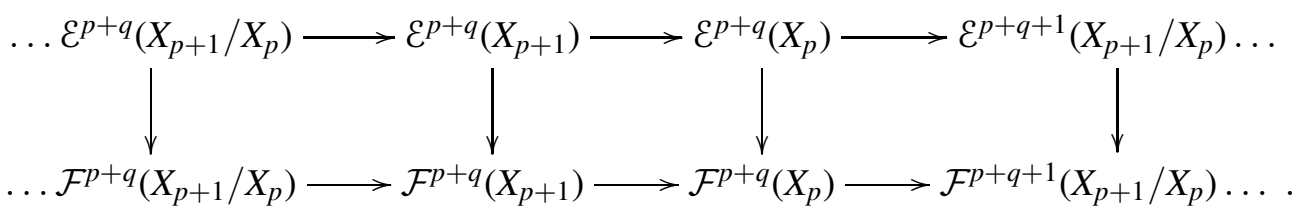

It follows immediately from the construction of the corresponding exact triangles that this morphism commutes with the differentials.

This now allows us to compare the topological and the smooth theories.

Corollary 15 Let $\mathcal{E}$ be a smooth spectrum and $\Pi \mathcal{E}$ be the underlying topological theory. Let $E_{n}$ and $F_{n}$ denote the spectral sequences corresponding to $\mathcal{E}$ and $\Pi \mathcal{E}$, respectively. The natural map $I: \mathcal{E} \rightarrow \Pi \mathcal{E}$ induces a morphism of classical AHSS's ${ }^{2}$

$$
I: E_{n}^{p, q} \rightarrow F_{n}^{p, q} .
$$

Remark 12 It is interesting to note that the smooth spectrum $\Pi \mathcal{E}$ is, by definition, locally constant. From the discussion around (2-1), this means that we have isomorphism

$$
\Pi E^{q}(U) \simeq \pi_{-q} \operatorname{Map}(U, \Pi \mathcal{E}) \simeq \pi_{-q} \operatorname{Map}(*, \Pi \mathcal{E}) \simeq \pi_{-q} \Pi \mathcal{E} \simeq \Pi \mathcal{E}^{q}(*)
$$

for every element of a good open cover (or higher intersection) $U$. This connects, via Borsuk's theorem mentioned in Remark 10 above, the "smooth AHSS for locally constant coefficients" with the classical AHSS: the locally constant coefficients see each (contractible) patch as a point, and hence by Borsuk's theorem they see our "Čech filteration" to be the classical $C W$-cell filteration.

From the construction of our smooth AHSS, we immediately get that the spectral sequence associated to the smooth spectrum is a refinement of the classical topological AHSS.

Corollary 16 The spectral sequence $F_{n}^{p, q}$ is precisely the AHSS for the cohomology theory $\Pi \mathcal{E}$.

\footnotetext{
${ }^{2}$ Here we have an unfortunate conflict of notation. We are using the same symbols for the pages in the spectral sequences for both the classical and the refined theories. We will aim to make the context explicit whenever a possible ambiguity arises.
} 
We now would like to apply the above machinery to differential cohomology theories. In particular, we note that for a differential function $\operatorname{spectrum} \operatorname{diff}(\mathcal{E}, \mathrm{ch})$, the natural map

$$
I: \operatorname{diff}(\mathcal{E}, \mathrm{ch}) \rightarrow \underline{\mathcal{E}},
$$

which strips the differential theory of the differential data and maps to the bare underlying theory, is precisely the map induced by the unit $I:$ id $\rightarrow \Pi$. In the above discussion, we observed that this map always induces a morphism of spectral sequences. Moreover, the target spectral sequence is exactly the AHSS for the underlying topological theory. One might hope to be able to use this map to compare the differentials in the refined theory with those differentials in the classical AHSS.

Unfortunately, this does not work in practice, as we will see when we discuss applications in Sec. 4. The core issue is that the spectral sequence for the refined theory usually ends up shifted with respect to the classical AHSS. As a consequence, the nonzero terms in each sequence are interlaced with respect to one another and the map $I$ ends up killing all the nonzero terms. This, in turn, stems from the appearance of the Bockstein map (which raises degree by 1) in the differential cohomology diagram.

However, there is often a different map between the lower quadrants of the the two spectral sequence corresponding to $\operatorname{diff}(\mathcal{E}, \mathrm{ch})$ and $\mathcal{E}$, which lowers the degree as to match the corresponding nonzero entries. This map is related to the so-called boundary map between spectral sequences studied in [Mi81]. The next proposition concerns this map and will be essential for comparing the differentials in the refined theory to those of the classical theory.

Proposition 17 (i) Let $\mathcal{E}$ be a spectrum such that $\pi_{*}(\mathcal{E})$ is concentrated in degrees which are a multiple of some integer $n \geq 2$ (e.g. K-theory, Morava K-theory). Suppose, moreover, that $\pi_{*}(\mathcal{E})$ is projective in those degrees. Then the sequence of spectra

$$
\mathcal{E} \rightarrow \mathcal{E} \wedge M \mathbb{R} \rightarrow \mathcal{E} \wedge M U(1) \stackrel{\beta_{\mathcal{E}}}{\longrightarrow} \Sigma \mathcal{E},
$$

induces a short exact sequence on coefficients

$$
0 \rightarrow \pi_{*}(\mathcal{E}) \rightarrow \pi_{*}(\mathcal{E}) \otimes \mathbb{R} \rightarrow \pi_{*}(\mathcal{E}) \otimes U(1) \rightarrow 0 .
$$

(ii) Let $\beta$ denote the connecting homomorphism (i.e. the Bockstein) for the coefficient sequence (3-4). Let $E_{n}^{p, q}$ denote the spectral sequence corresponding to $\Sigma^{-1} \mathcal{E} \wedge M U(1)$ and let $F_{n}^{p, q}$ denote the spectral sequence corresponding to $\mathcal{E}$. Then

$$
\beta: E_{n}^{p, q} \rightarrow F_{n}^{p, q}
$$

induces a morphism of spectral sequences of rank 2 . 
Proof. Consider the long Bockstein sequence

$$
\ldots \mathcal{E} \stackrel{r}{\longrightarrow} \mathcal{E} \wedge M \mathbb{R} \stackrel{e}{\longrightarrow} \mathcal{E} \wedge M U(1) \stackrel{\beta_{\mathcal{E}}}{\longrightarrow} \Sigma \mathcal{E} \ldots
$$

induced by the cofiber sequence

$$
\mathbb{S} \rightarrow M \mathbb{R} \rightarrow M U(1) .
$$

Fix a manifold $M$ and let $X_{p}$ denote the $p$-level of the Čech filtration. Now each spectrum in the above sequence has a long exact sequence induced be the cofiber sequences

$$
X_{p-1} \rightarrow X_{p} \rightarrow X_{p} / X_{p-1}
$$

from which one builds the exact couple for for the corresponding spectral sequence. Using the properties of $\pi_{*}(\mathcal{E})$ along with this sequence, we can fit the long exact sequences into a diagram

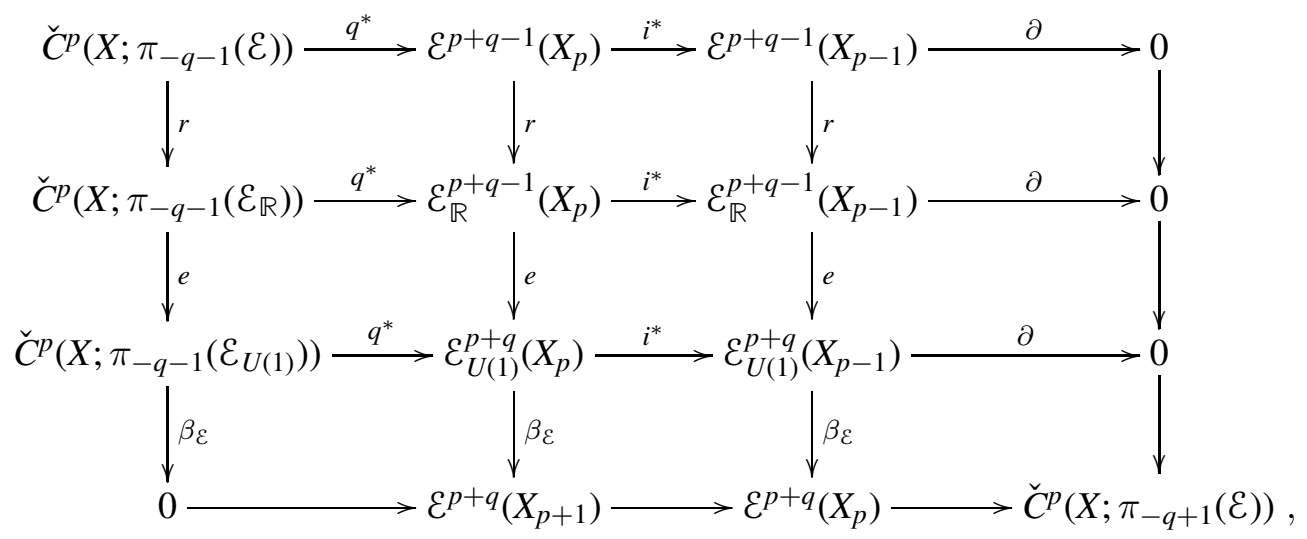

where both the rows and columns are part of exact sequences and $\check{C}^{p}(X ; A)$ denotes the group of Čech $p$-cochains with coefficients in $A$. Since everything commutes, this induces a correponding short exact sequence of $E_{1}$ pages. At each $(p, q)$-entry this sequence is given by

$$
0 \rightarrow C^{p}\left(X ; \pi_{-q}(\mathcal{E})\right) \rightarrow C^{p}\left(X ; \pi_{-q}(\mathcal{E}) \otimes \mathbb{R}\right) \rightarrow C^{p}\left(X ; \pi_{-q}(\mathcal{E}) \otimes U(1)\right) \rightarrow 0 .
$$

Since the differentials on the $E_{1}$ page are precisely the Čech differentials, the construction of the Bockstein map in Čeach cohomology will produce a map of $E_{2}$-pages

$$
\beta: H^{p}\left(X ; \pi_{-q}(\mathcal{E}) \otimes U(1)\right) \rightarrow H^{p+1}\left(X ; \pi_{-q}(\mathcal{E})\right) .
$$

We need to show that this map commutes with the differential. Choose a representative $x$ of a class in $H^{p}\left(X ; \pi_{-q}(\mathcal{E}) \otimes U(1)\right)$. By definition, $y=\beta(x)$ is a class such that 
$r(y)=\delta(\bar{x})$, where $\bar{x}$ is such that $e(\bar{x})=x$. Then

$$
r\left(d_{2} y\right)=d_{2} r(y)=d_{2} \delta(\bar{x}),
$$

We want to show that there is a lift $z$ of $d_{2} x$ such that $\delta(z)=d_{2} \delta(\bar{x})$. Indeed, if this is the case, then $d_{2} y$ represents $\beta\left(d_{2} x\right)$ and we are done.

To construct $z$, recall that $d_{2} x$ is defined by first pulling back by the quotient $q$, which lies in the image of the map induced by the inclusion $i: X_{p} \hookrightarrow X_{p+1}$, and then applying the boundary to an element of the preimage. Let $w$ be such that

$$
i^{*}(w)=q^{*}(x) .
$$

Chasing the diagram

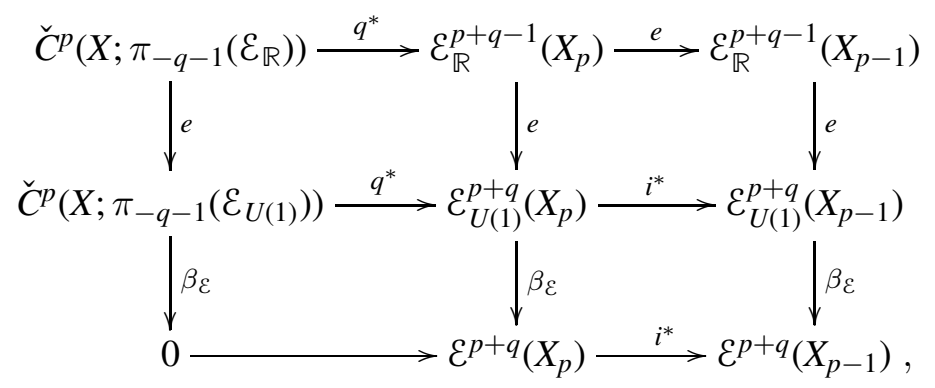

we see that $0=\beta_{\varepsilon} q^{*}(x)=\beta_{\varepsilon} i^{*}(w)=i^{*}\left(\beta_{\mathcal{E}} w\right)$. By exactness of the rows, this implies that $\beta_{\mathcal{E}} w=0$. Therefore, there is a class $\bar{w} \in \mathcal{E}_{\mathbb{R}}^{p+q+1}\left(X_{p+1}\right)$ such that $e(\bar{w})=w$.

Now, by definition of the differential, we have

$$
e(\partial \bar{w})=\partial(e(\bar{w}))=\partial w=d_{2} x
$$

and $z:=\partial \bar{w}$ is a lift of $d_{2} x$. Using the fact that $\delta=d_{1}=\partial q^{*}$, we have

$$
\delta(z)=\delta(\partial \bar{w})=\partial\left(q^{*} \partial \bar{w}\right) .
$$

By exactness, we have

$$
i^{*}\left(q^{*} \partial \bar{w}\right)=0=q^{*} \partial q^{*}(\bar{x})=q^{*}(\delta(\bar{x})),
$$

and it follows from the definition that $\delta(z)=d_{2}(\delta(\bar{x}))$.

To show that $H^{*}(\beta)$ commutes with the higher differentials, we proceed by induction. The above proves the base case. Suppose $\beta$ induces a map $H_{n}(\beta)$ on $E_{n}$ which commutes with $d_{n}$. Then $H^{n}(\beta)$ induces a well defined map $H^{n+1}(\beta)$ on the $E_{n+1}$ page. Let $x \in \bigcap_{i=1}^{n} \operatorname{ker}\left(d_{n+1}\right)$ be a representative of a class on the $E_{n}$ page. Then be definition, $H^{n+1}(\beta)(x)=\beta(x)$ and the exact same argument as before (replacing $d_{2}$ 
with $d_{n+1}$ ), gives the result.

Having done the heavy lifting in the above proposition, we will now apply this to straightforwardly relate the differentials of the refined theory to those of the underlying topological theory. This will use an explicit alternative to the map $I$, along the lines of the discussion just before the statement of the above proposition.

Theorem 18 (Refinement of differentials) Let $\mathcal{E}$ be a spectrum satisfying the properties of Proposition 17 and let diff $(\mathcal{E}, \mathrm{ch})$ be a differential function spectrum refining $\mathcal{E}$. Let $E_{n}$ and $F_{n}$ denote the smooth AHSS's corresponding to $\operatorname{diff}(\mathcal{E}, \mathrm{ch})$ and $\mathcal{E}$, respectively. Then the Bockstein $\beta$ defines a rank 2 morphism of fourth quadrant spectral sequences

$$
\beta: E_{n}^{p, q} \rightarrow F_{n}^{p, q}, q<0
$$

Proof. Recall that for $q<0$, Proposition 4 implies that $\operatorname{diff}(\mathcal{E}, \mathrm{ch})^{q}(M) \simeq \mathcal{E}_{U(1)}^{q-1}(M)$. The claim then follows from the previous proposition.

\subsection{Product structure and the differentials}

Let $\mathcal{E}$ be an $E_{\infty}$ ring spectrum. Then the associative graded-commutative product on $\mathcal{E}^{*}$ induces a product (associative and graded-commutative) on the refinement $\operatorname{diff}\left(\Sigma^{n} \mathcal{E}, \mathrm{ch}\right)^{*}$, that is, a map

$$
\cup: \operatorname{diff}\left(\Sigma^{n} \mathcal{E}, \mathrm{ch}\right)^{k} \otimes \operatorname{diff}\left(\Sigma^{m} \mathcal{E}, \mathrm{ch}\right)^{j} \longrightarrow \operatorname{diff}\left(\Sigma^{n+m} \mathcal{E}, \mathrm{ch}\right)^{k+j}
$$

(see [Bu12] [Up15]). The goal of this section will be to establish the following very useful property, in analogy with the classical case.

Proposition 19 (Compatibility with products) The product

$$
\cup: \operatorname{diff}\left(\Sigma^{n} \mathcal{E}, \mathrm{ch}\right)^{k} \otimes \operatorname{diff}\left(\Sigma^{m} \mathcal{E}, \mathrm{ch}\right)^{j} \rightarrow \operatorname{diff}\left(\sum^{n+m} \mathcal{E}, \mathrm{ch}\right)^{k+j}
$$

induces a morphism of spectral sequences

$$
\cup: E_{*}(n) \times E_{*}(m) \rightarrow E_{*}(n+m) .
$$

Moreover, the differentials satisfy the Liebniz rule

$$
d(x y)=d(x) y+(-1)^{p+q} x d(y) .
$$


Let us first work out what the cup product pairing is on the $E_{1}$-page. Recall from the construction of the spectral sequence that the $E_{1}^{p, q}$ is given by

$$
E_{1}^{p, q}=\bigoplus_{\alpha_{0} \ldots \alpha_{p}} \operatorname{diff}\left(\Sigma^{n} \mathcal{E}, \mathrm{ch}\right)^{q}\left(U_{\alpha_{0} \ldots \alpha_{p}}\right) \simeq \check{C}^{p}\left(M ; \operatorname{diff}\left(\Sigma^{n} \mathcal{E}, \mathrm{ch}\right)^{q}\right) .
$$

Using the product (3-5), we get a cross product map

$$
\begin{aligned}
\times: \bigoplus_{\alpha_{0} \ldots \alpha_{p}} \operatorname{diff}\left(\Sigma^{n} \mathcal{E}, \mathrm{ch}\right)^{q}\left(U_{\alpha_{0} \ldots \alpha_{p}}\right) & \times \bigoplus_{\alpha_{0} \ldots \alpha_{r}} \operatorname{diff}\left(\Sigma^{m} \mathcal{E}, \mathrm{ch}\right)^{t}\left(U_{\alpha_{0} \ldots \alpha_{r}}\right) \rightarrow \\
& \rightarrow \bigoplus_{\alpha_{0} \ldots \alpha_{p}} \bigoplus_{\alpha_{0} \ldots \alpha_{r}} \operatorname{diff}\left(\Sigma^{n+m} \mathcal{E}, \mathrm{ch}\right)^{q+t}\left(U_{\alpha_{0} \ldots \alpha_{p}} \times U_{\alpha_{0} \ldots \alpha_{r}}\right) .
\end{aligned}
$$

We also have an isomorphism

$$
\begin{aligned}
\bigoplus_{\alpha_{0} \ldots \alpha_{s}} \operatorname{diff}\left(\Sigma^{n+m} \mathcal{E}, \operatorname{ch}\right)^{q+t}\left((U \times U)_{\alpha_{0} \ldots \alpha_{s}}\right) & \simeq \operatorname{diff}\left(\Sigma^{n+m} \mathcal{E}, \operatorname{ch}\right)^{q+t}\left(\bigvee_{\alpha_{0} \ldots \alpha_{s}}(U \times U)_{\alpha_{0} \ldots \alpha_{s}}\right) \\
& \simeq \operatorname{diff}\left(\Sigma^{n+m} \mathcal{E}, \operatorname{ch}\right)^{q+t}\left(\bigvee_{\alpha_{0} \ldots \alpha_{p}} \bigvee_{\alpha_{0} \ldots \alpha_{r}} \bigvee_{p+r=s} U_{\alpha_{0} \ldots \alpha_{p}} \times U_{\alpha_{0} \ldots \alpha_{r}}\right) \\
& \simeq \bigoplus_{\alpha_{0} \ldots \alpha_{p} \alpha_{0} \ldots \alpha_{r}} \bigoplus_{p+r=s} \operatorname{diff}\left(\Sigma^{n+m} \mathcal{E}, \mathrm{ch}\right)^{q+t}\left(U_{\alpha_{0} \ldots \alpha_{p}} \times U_{\alpha_{0} \ldots \alpha_{r}}\right)
\end{aligned}
$$

given by decomposing the product of the cover $\left\{U_{\alpha}\right\}$ with itself. Finally, we can pullback by the diagonal map

$$
\begin{array}{r}
\Delta^{*}: \bigoplus_{\alpha_{0} \ldots \alpha_{s}} \operatorname{diff}\left(\Sigma^{n+m} \mathcal{E}, \mathrm{ch}\right)^{q+t}\left((U \times U)_{\alpha_{0} \ldots \alpha_{s}}\right) \rightarrow \bigoplus_{\alpha_{0} \ldots \alpha_{s}} \operatorname{diff}\left(\Sigma^{n+m} \mathcal{E}, \mathrm{ch}\right)^{q+t}\left(U_{\alpha_{0} \ldots \alpha_{s}}\right) \simeq \\
\simeq \check{C}^{p+r}\left(M ; \operatorname{diff}\left(\Sigma^{n+m} \mathcal{E}, \mathrm{ch}\right)^{q+t}\right) .
\end{array}
$$

The cup product on the $E_{1}$-page is defined by the composite map $\Delta^{*} \times$.

Lemma 20 The differential $d_{1}$ on the $E_{1}$-page satisfies the Leibniz rule.

Proof. The construction of the cup product on the $E_{1}$-page is precisely the cup product structure for Čech-cohomology. The Čech differential satisfies the Leibniz rule and this is precisely $d_{1}$, by construction.

We are now ready to prove Proposition 19. 
Proof. The proof follows by induction on the pages of the spectral sequence. The base case is satisfied by Lemma 20. Now suppose we have a cup product map

$$
\cup: E(n)_{k} \times E(n)_{k} \rightarrow E(n+m)_{k},
$$

such that $d_{k}$ satisfies Leibniz. By definition, we have

$$
E(n)_{k+1}^{p, q}=\frac{\operatorname{ker}\left(d_{k}: E(n)_{k}^{p, q} \rightarrow E(n)_{k}^{p+k, q+k-1}\right)}{\operatorname{im}\left(d_{k}: E(n)^{p-k, q-k+1} \rightarrow E(n)^{p, q}\right)},
$$

and we define the cup product

$$
\cup: E(n)_{k+1}^{p, q} \times E(m)_{k+1}^{r, s} \rightarrow E(n+m)_{k+1}^{p+r, q+s}
$$

by restricting to elements in the kernel of $d_{k}$. The product is well defined since $d_{k}$ satisfies the Leibniz rule. At this stage the problem looks formally like the classical problem. Hence, analogously to the classical discussion in [Ha02], it is tedious but straightforward to show that $d_{k+1}$ also satisfies the Leibniz rule.

\section{Applications to differential cohomology theories}

In this section we would like to apply the spectral sequence constructed in the previous section to various differential cohomology theories. The construction is general enough to apply to any structured cohomology theory whose coefficients are known. We will explicitly emphasize three main examples. The first two are to known theories, namely ordinary differential cohomology and differential K-theory. We take this opportunity to explicitly develop the third theory, which is differential Morava K-theory and then apply our smooth AHSS construction to it.

\subsection{Ordinary differential cohomology theory}

We begin by recovering the usual hypercohomology spectral sequence for the Deligne complex (see [Br93], [EV92] Appendix) using our methods. We will first look at manifolds, then products of these, and then more generally to smooth fiber bundles.

Let us consider the smooth spectrum $\operatorname{diff}\left(\sum^{n} H \mathbb{Z}\right.$, ch) representing differential cohomology in degree $n$. We would like to see what our smooth AHSS gives in this case. We recall that $\operatorname{diff}\left(\Sigma^{n} H \mathbb{Z}, \mathrm{ch}\right)$ is represented by Deligne cohomology of the sheaf of chain complexes $\mathbb{Z}_{\mathcal{D}}^{\infty}(n)$ via the Eilenberg-MacLane functor $H: S h_{\infty}\left(C_{\text {artsp; }} \mathrm{Ch}\right) \rightarrow$ 
$S h_{\infty}($ CartSp; Sp) (expressions (2-6)). It follows from the general properties of this functor that the homotopy groups are given by

$$
\pi_{k} \operatorname{diff}\left(\Sigma^{n} H \mathbb{Z}, \mathrm{ch}\right) \simeq H_{k} \mathbb{Z}_{\mathcal{D}}^{\infty}(n) .
$$

In this case we have the immediate corollary to Theorem 11.

Corollary 21 The spectral sequence for Deligne cohomology takes the form

$$
E_{2}^{p, q}=H^{p}\left(X ; H_{-q} \mathbb{Z}_{\mathcal{D}}^{\infty}(n)\right) \Rightarrow H^{p+q}\left(X ; \mathbb{Z}_{\mathcal{D}}^{\infty}(n)\right),
$$

which is essentially the hypercohomology spectral sequence for the Deligne complex, but shifted as a fourth quadrant spectral sequence.

For the sake of completeness, we work out this spectral sequence and recover the differential cohomology diamond (2-2) from the sequence. This will help to illustrate how the general spectral sequence behaves and how it can be used to calculate general differential cohomology groups.

Now over the site of Cartesian spaces, the Poincare Lemma implies that we have an isomorphism of presheaves $d: \Omega^{n-1} / \mathrm{im}(\mathrm{d}) \stackrel{\simeq}{\rightarrow} \Omega_{\mathrm{cl}}^{n}$. Since $\Omega_{\mathrm{cl}}^{n}$ is a sheaf over the site of smooth manifolds, the gluing condition allows us to calculate the relevant terms on the $E_{2}$-page of the spectral sequence:

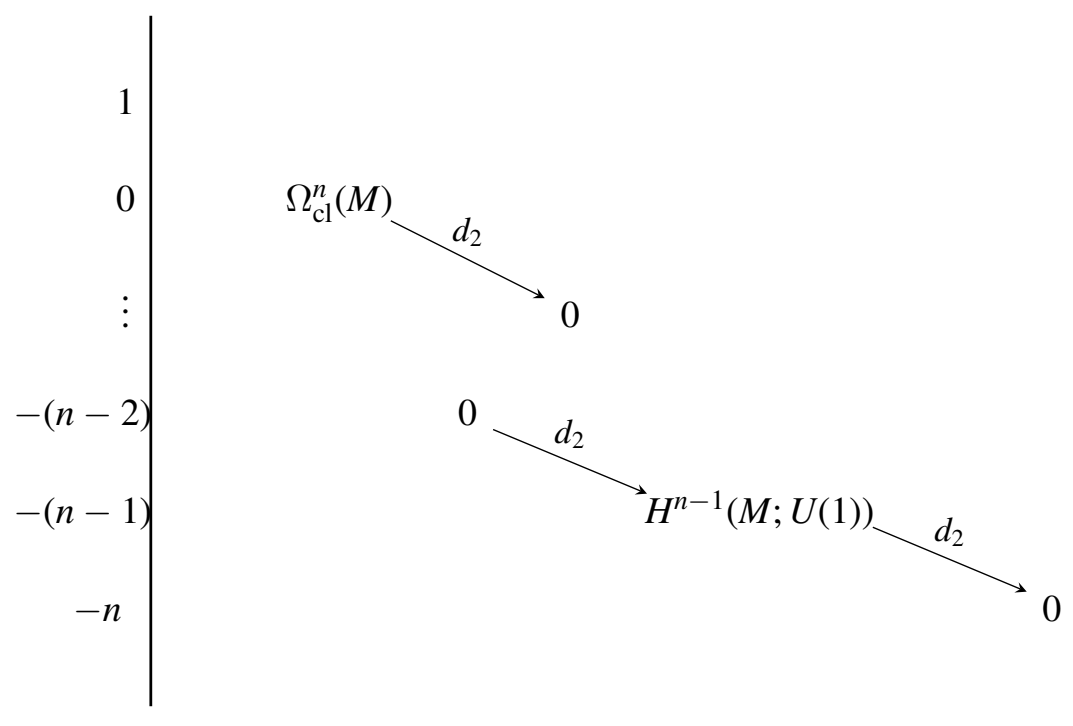


The term $H^{n-1}(M ; U(1))$ will survive to the $E_{\infty}$-page and we have an isomorphism

$$
H^{n-1}(M ; U(1)) \simeq \frac{F_{n-1} \widehat{H}^{n}(M ; \mathbb{Z})}{F_{n} \widehat{H}^{n}(M ; \mathbb{Z})} .
$$

In fact, it is not hard to see that the definition of the filtration gives $F_{n} \widehat{H}^{n}(M ; \mathbb{Z}) \simeq 0$ and we have an injection

$$
H^{n-1}(M ; U(1)) \simeq F_{n-1} \widehat{H}^{n}(M ; \mathbb{Z}) \hookrightarrow \widehat{H}^{n}(M ; \mathbb{Z}) .
$$

On the $E_{n}$-page we get one possibly nonzero differential

$$
d_{n}: \Omega^{n}(M)_{\mathrm{cl}} \rightarrow H^{n}(M ; U(1)) .
$$

Proposition 22 The differential $d_{n}$ for the AHSS for Deligne cohomology can be identified with the composition

$$
\Omega_{\mathrm{cl}}^{n}(M) \rightarrow H_{\mathrm{dR}}^{n}(M) \stackrel{\int_{\Delta^{n}}}{\longrightarrow} H^{n}(M ; \mathbb{R}) \stackrel{\exp }{\longrightarrow} H^{n}(M ; U(1)),
$$

and the kernel is precisely those forms which have integral periods.

Proof. We will unpack the definition of the differential in the AHSS in detail. This in turn will require unpacking the connecting homomorphism in the Deligne model of ordinary differential cohomology (see [Br93]). Denote by $X_{p}$ the Čech filtration, and let

$$
\partial: \operatorname{diff}\left(\Sigma^{n} H \mathbb{Z}, \mathrm{ch}\right)^{q}\left(X_{p}\right) \rightarrow \operatorname{diff}\left(\Sigma^{n} H \mathbb{Z}, \mathrm{ch}\right)^{q+1}\left(X_{p+1} / X_{p}\right)
$$

denote the connecting homomorphism in the long exact sequence associated to the cofiber sequence $X_{p} \hookrightarrow X_{p+1} \rightarrow X_{p+1} / X_{p}$ in the usual way. In what follows, we will denote Čech-Deligne cochains on the $p$-th level of the filtration $X_{p}$ as a $p$-tuple

$$
\left(z_{0}, z_{1}, \ldots, z_{p}\right) \in \widehat{C}^{q}\left(X_{p}\right),
$$

where $z_{i}$ is a $(q-i)$-form defined on $i$-fold intersections.

Now, by definition, $d_{n}: E_{n}^{0,0} \rightarrow E_{n}^{n, 0}$ is given by $d_{n}=\partial\left(j^{*}\right)^{-1}$, where $\left(j^{*}\right)^{-1}$ denotes a choice of element in the preimage of the restriction $j^{*}$ induced by ${ }^{3} j: X_{0} \hookrightarrow X_{n-1}$. Since we have $d_{k}=0$ for $k<n$, the differential $d_{n}$ is defined on all elements $z \in \Omega_{\mathrm{cl}}^{n}(M)$. Let $g_{0}$ be a locally defined $(n-1)$-form trivializing $z$. Then we can choose $\left(j^{*}\right)^{-1} z$ to be the Čech-Deligne cocycle

$$
\left(j^{*}\right)^{-1} z=\underbrace{\left(g_{0}, g_{1}, g_{2}, \ldots, g_{n-2}\right)}_{n-1} \in \widehat{C}^{0}\left(X_{n-1}\right),
$$

\footnotetext{
${ }^{3}$ Note that the differential only takes this form at the $(0,0)$-entry. In general, the differential formed from the $n$-th derived couple will be more complicated
} 
where each $g_{k}$ is a $(n-k-1)$-form satisfying the cocycle condition $\delta\left(g_{k}\right)=$ $(-1)^{k} d g_{k+1}$. To see where the boundary map takes this element, let $y$ be a ČechDeligne cochain given by

$$
y=\underbrace{\left.\left(g_{0}, g_{1}, g_{2}, \ldots, g_{n-2}, \exp \left(2 \pi i g_{n-1}\right)\right)\right)}_{n} \in \widehat{C}^{0}\left(X_{n}\right),
$$

where $g_{n-1}$ is any smooth $\mathbb{R}$-valued function satisfying ${ }^{4} d\left(g_{n-1}\right)=(-1)^{n-1} \delta\left(g_{n-2}\right)$. Now $y$ is not Čech-Deligne closed in general since

$$
D y=\left(d+(-1)^{n-1} \delta\right) y=\left(0,0, \ldots, \exp \left((-1)^{n-1} 2 \pi i \cdot \delta\left(g_{n-1}\right)\right)\right)
$$

and $g_{n-1}$ may not satisfy the cocycle condition $\delta\left(g^{n-1}\right)=0$. However, by the Čechde Rham isomorphism (see for example [BT82]), this element in the Čech-de Rham double complex is isomorphic to an $\mathbb{R}$-valued Čech cocycle on $n$-fold intersections. Explicitly, there is a constant $\mathbb{R}$-valued cocycle $r_{n}$ such that $\delta\left(g^{n-1}\right)=r_{n}$. It follows from the Cech-singular isomorphism and the singular-de Rham isomorphism that the class of $r_{n}$ can be represented by the singular cocycle given by the pairing $\int_{\sigma} z$ for any cycle $\sigma$ in $M$. Since the class $\int_{\sigma} z$ was just an unraveling of the boundary $\partial\left(\left(j^{*}\right)^{-1} z\right)$, we have proved the claim.

In the next section, we will need to make use of a differential refinement of the Chern character. To this end, we briefly discuss differential cohomology with rational coefficients $\widehat{H}^{n}(-; \mathbb{Q})$. These groups are obtained via the differential function spectra $\operatorname{diff}\left(\Sigma^{n} H \mathbb{Q}, \mathrm{ch}\right)$ which fits into the homotopy cartesian square

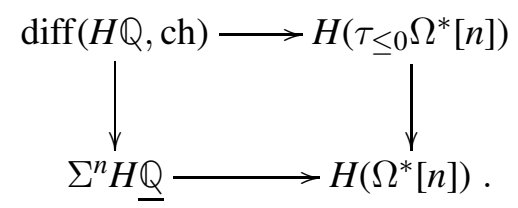

As a consequence of Proposition 4, the cohomology groups with values in this spectrum are calculated as

$$
\operatorname{diff}\left(\Sigma^{n} H \mathbb{Q}, \operatorname{ch}\right)^{q}(M)=\left\{\begin{array}{cc}
H^{n+q}(M) & q>0, \\
\widehat{H}^{n}(M ; \mathbb{Q}) & q=0 \\
H^{n-1+q}(M ; \mathbb{R} / \mathbb{Q}) & q<0 .
\end{array}\right.
$$

\footnotetext{
${ }^{4}$ Note that this cocycle condition is necessary for $y$ to be an lift of $\left(j^{*}\right)^{-1} z$ to the $n$-level of the filtration
} 
The explicit calculation of the differential in Proposition 22 can be easily modified to get the following. ${ }^{5}$

Proposition 23 The differential $d_{n}$ on the $E_{n}$-page for the AHSS spectral sequence for $\operatorname{diff}\left(\Sigma^{n} H \mathbb{Q}, \mathrm{ch}\right)$ is given by

$$
\Omega_{\mathrm{cl}}^{n}(M) \rightarrow H_{\mathrm{dR}}^{n}(M) \stackrel{\int_{\Delta^{n}}}{\longrightarrow} H^{n}(M ; \mathbb{R}) \longrightarrow H^{n}(M ; \mathbb{Q} / \mathbb{Z}),
$$

and the kernel is precisely those forms which have rational periods.

We will make use of this result when we discuss the differentials in smooth K-theory in the next section. For now, from Proposition 22, we immediately get the following characterization of closed forms with integral periods and forms with rational periods using our smooth AHSS.

Corollary 24 (i) The group of closed forms with integral periods on a manifold $M$ is given by

$$
\Omega_{\mathrm{cl}, \mathbb{Z}}^{n}(M) \simeq \frac{\widehat{H}^{n}(M ; \mathbb{Z})}{F_{1} \widehat{H}^{n}(M ; \mathbb{Z})}
$$

(ii) The group of closed forms with rational periods on a manifold $M$ is given by

$$
\Omega_{\mathrm{cl}, \mathbb{Q}}^{n}(M) \simeq \frac{\widehat{H}^{n}(M ; \mathbb{Q})}{F_{1} \widehat{H}^{n}(M ; \mathbb{Q})} .
$$

\subsection{Differential $K$-theory}

In this section we examine the smooth AHSS for the differential function spectrum $\operatorname{diff}(K, \mathrm{ch})$, corresponding to complex $K$-theory. Proposition 4 allows us to calculate the cohomology groups on a paracompact manifold $M$ as (see [Lo94] [BS09] [SS08] [FL10])

$$
\operatorname{diff}(K, \operatorname{ch})^{q}(M)=\left\{\begin{array}{cc}
K^{q}(M), & q>0, \\
\widehat{K}^{0}(M), & q=0, \\
K_{U(1)}^{q}(M), & q<0 .
\end{array}\right.
$$

\footnotetext{
${ }^{5}$ The exact argument in the proof of proposition 22 applies, with $\mathbb{R} / \mathbb{Q}$ in place of $\mathbb{R} / \mathbb{Z} \simeq$ $U(1)$.
} 
Now both groups $K$ and $K_{U(1)}$ are periodic. Indeed, $K_{U(1)}(M)$ fits into an exact sequence

$$
\ldots \rightarrow K^{-1}(M) \otimes \mathbb{R} \rightarrow K_{U(1)}^{-1}(M) \rightarrow K(M) \rightarrow K(M) \otimes \mathbb{R} \rightarrow \ldots .
$$

Consequently, the periodicity of both integral and rational $K$-theory, along with an application of the Five Lemma, imply that $K_{U(1)}$ is 2-periodic. In particular, we have

$$
K_{U(1)}^{2 q}(*) \simeq U(1) \quad \text { and } \quad K_{U(1)}^{2 q+1}(*) \simeq 0, \quad q \in \mathbb{Z} .
$$

Given the correspondence (4-2), we see that for a contractible open set $U$, we have an isomorphism

$$
\operatorname{diff}(K, \operatorname{ch})^{2 q+1}(U) \simeq K_{U(1)}^{2 q}(*) \simeq U(1)
$$

for $q<0$. For degree 0 , the differential cohomology diamond in this case takes the form

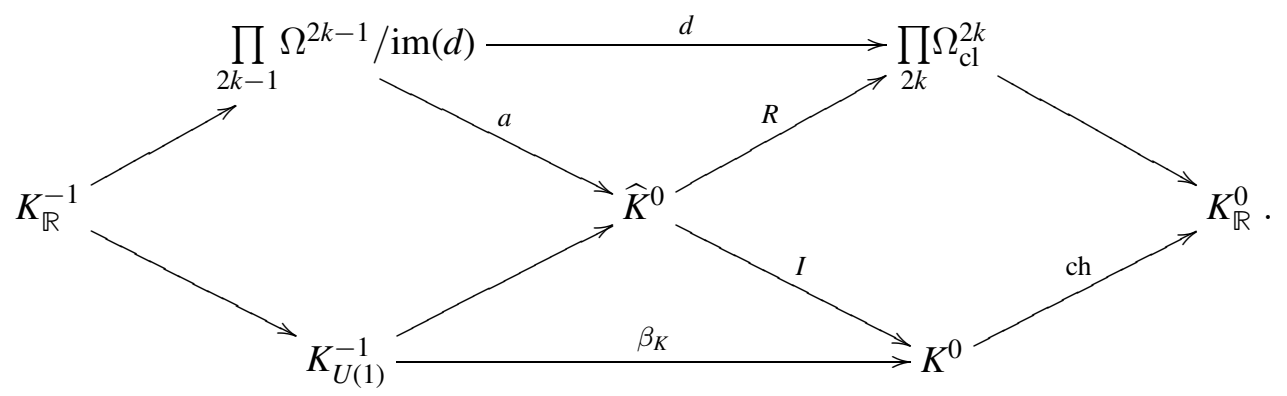

This implies that for a contractible open set $U$, differential K-theory $\widehat{K}^{0}(U)$ fits into the short exact sequence

$$
0 \rightarrow \prod_{2 k-1} \Omega^{2 k-1} / \operatorname{im}(d)(U) \rightarrow \widehat{K}^{0}(U) \rightarrow \mathbb{Z} \rightarrow 0 .
$$

Hence, over the site of Cartesian spaces, we have a naturally split short exact sequence of presheaves

$$
0 \rightarrow \prod_{2 k-1} \Omega^{2 k-1} / \operatorname{im}(d) \rightarrow \widehat{K}^{0} \rightarrow \underline{\mathbb{Z}} \rightarrow 0 .
$$

Over that site, the presheaf on the left hand side is actually a sheaf and is naturally isomorphic (by Poincaré lemma) to the sheaf $\prod_{2 k} \Omega_{\mathrm{cl}}^{2 k}$. We therefore make the identification

$$
\widehat{K}^{0} \simeq \prod_{2 k} \Omega_{\mathrm{cl}}^{2 k} \oplus \underline{\mathbb{Z}}
$$

Remark 13 It is important to note that the identification (4-3) is only true on the site 
of Cartesian spaces, which is to say that it holds only locally. On the site of smooth manifolds, this is of course not the case.

Next, since both $\Omega_{\mathrm{cl}}^{2 k}$ and $\mathbb{Z}$ are sheaves on the site of smooth manifolds, we can identify the degree 0 Čech cohomology with these coefficients with the value of this sheaf on $M$. Isolating the terms on the $E_{2}$-page which converge to $\widehat{K}^{0}(M)$, we get

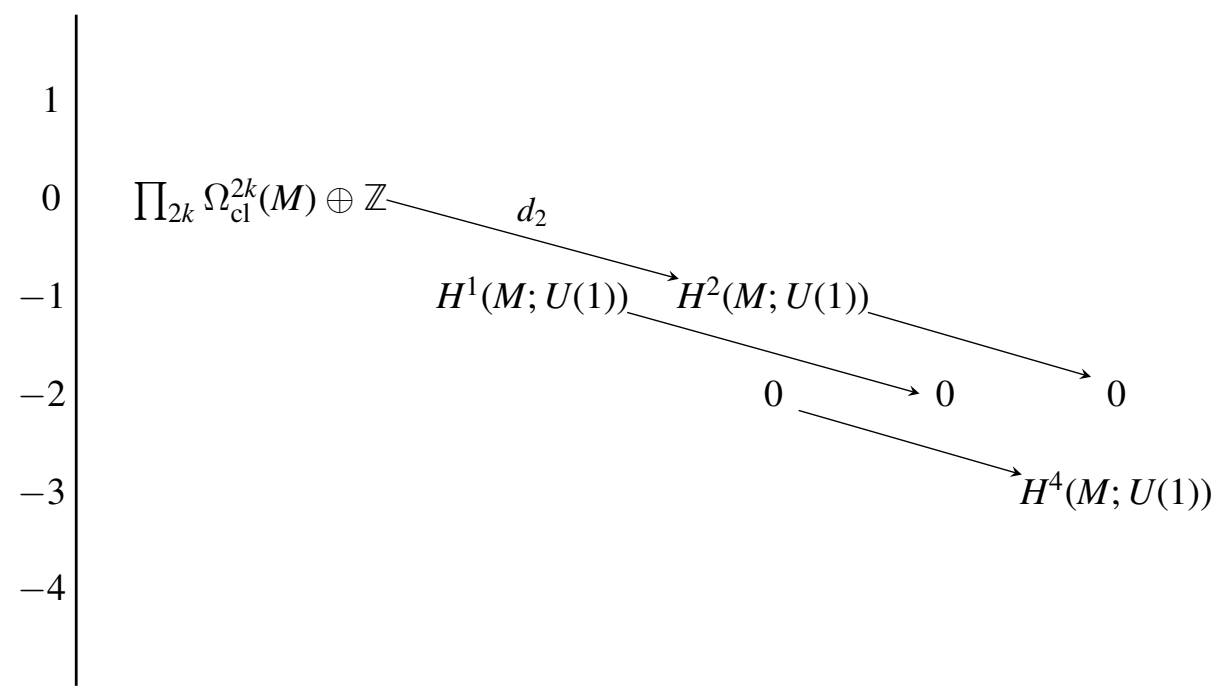

We see that all the differentials are zero except for the map labelled $d_{2}$ above. On the 
$E_{3}$-page we get

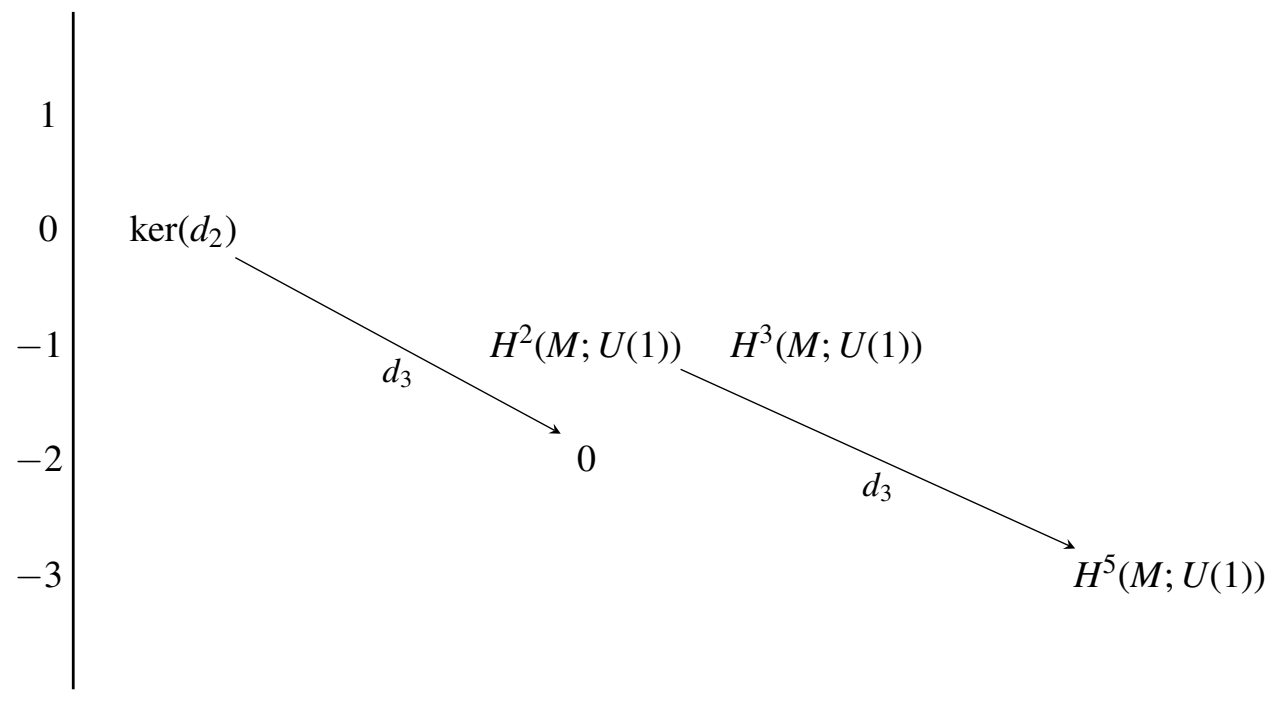

The higher pages will fall into cases depending on the parity. We observe that for each even page $E_{2 m}$, there is one non-zero differential given by $d_{2 m}$. For the odd pages the differentials are given by an odd-degree $U(1)$-cohomology operation.

Note that, in the diagrams, we are interested in the case $p+q=0$, corresponding to diagonal entries. Now $p \geq 0$, as the Čech filtrations are of non-negative degrees, which implies that $q \leq 0$. Hence the entries go down the diagonal. Our first goal will be to identify the even differentials $d_{2 m}$. In order to do this, let us recall that there is a differential Chern character map (see [Bu12] [Sc13]) which is stably given by a morphism of smooth spectra

$$
\widehat{\mathrm{ch}}: \operatorname{diff}(K, \mathrm{ch}) \rightarrow \prod_{2 k} \operatorname{diff}\left(\Sigma^{2 k} H \mathbb{Q}, \mathrm{ch}\right) .
$$

Post-composing this map with the projection $\mathrm{pr}_{2 m}$ onto the $2 m$-component gives a map of smooth spectra

$$
\operatorname{pr}_{2 m} \widehat{c h}: \operatorname{diff}(K, \mathrm{ch}) \rightarrow \operatorname{diff}\left(\Sigma^{2 m} H \mathbb{Q}, \mathrm{ch}\right) .
$$

Using this map, we can prove the following analogue of Proposition 23.

Proposition 25 The group of permanent cycles in bidegree $(0,0)$ in the AHSS for $\operatorname{diff}(K, \mathrm{ch})$ is a subgroup of even degree closed forms with rational periods. That is, 
we have

$$
E_{\infty}^{0,0} \subset \prod_{k} \Omega_{\mathrm{cl}, \mathbb{Q}}^{2 k}(M) \oplus \mathbb{Z}
$$

Proof. We prove by induction on the even pages of the spectral sequence that, for all $n, E_{2 n}^{0,0}$ must be a subgroup of ${ }^{6}$

$$
\prod_{2 k \leq 2 n} \Omega_{\mathrm{cl}, \mathbb{Q}}^{2 k}(M) \oplus \prod_{2 k>2 n} \Omega_{\mathrm{cl}}^{2 k}(M) \oplus \mathbb{Z} .
$$

For the base case, observe that the map $\mathrm{pr}_{2} \widehat{c h}$ induces a rank 1 morphism of AHSS's and therefore commutes with $d_{2}$. It is straightforward to check, using the definitions, that this leads to the commutative diagram

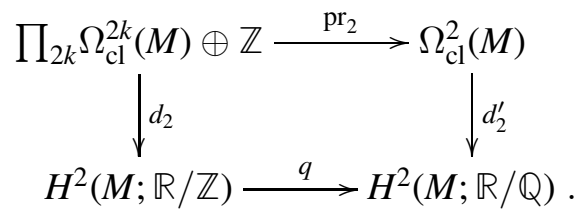

By Proposition 22, we see that the kernel of $d_{2}$ must be a subgroup of $\Omega_{\mathrm{cl}, \mathbb{Q}}^{2}(M) \oplus$ $\prod_{2 k>2} \Omega^{2 k}(M) \oplus \mathbb{Z}$.

Now suppose the claim is true for $d_{2 n}$. Again, we have that $\mathrm{pr}_{2 n+2} \widehat{\mathrm{ch}}$ commutes with $d_{2 n+2}$ and we have a commutative diagram

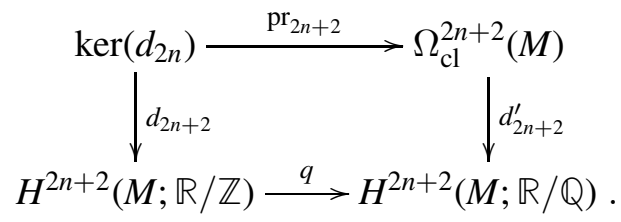

By the induction hypothesis,

$$
\operatorname{ker}\left(d_{2 n}\right) \subset \prod_{2 k \leq 2 n} \Omega_{\mathrm{cl}, \mathbb{Q}}^{2 k}(M) \oplus \prod_{2 k>n} \Omega_{\mathrm{cl}}^{2 k}(M) \oplus \mathbb{Z},
$$

and the kernel of $d_{2 n+2}$ is as claimed.

We now turn to the first odd differential $d_{3}$. Recall that $\beta$ and $\tilde{\beta}$ denote the Bockstein homomorphisms corresponding to the sequences $0 \rightarrow \mathbb{Z} \rightarrow \mathbb{R} \stackrel{\exp }{\longrightarrow} U(1) \rightarrow 0$ and

\footnotetext{
${ }^{6}$ The differential is 0 for the odd pages, and so no generality is lost by restricting to the even pages.
} 
$0 \rightarrow \mathbb{Z} \rightarrow \mathbb{Z} \rightarrow \mathbb{Z} / 2 \rightarrow 0$, respectively. We still also denote by $\Gamma_{2}: H^{n}(-; \mathbb{Z} / 2) \rightarrow$ $H^{n}(-, U(1))$ the map induced by the representation of $\mathbb{Z} / 2$ as the square roots of unity and $\rho_{2}: \mathbb{Z} \rightarrow \mathbb{Z} / 2$ as the $\bmod 2$ reduction.

Proposition 26 (Degree three differential) The first odd-degree differential in the AHSS for differential K-theory is given by

$$
d_{3}=\left\{\begin{array}{cc}
\widehat{S q}^{3}:=\Gamma_{2} S q^{2} \rho_{2} \beta, & q<0, \\
S q_{\mathbb{Z}}^{3}:=\tilde{\beta} S q^{2} \rho_{2}, & q>0, \\
0, & q=0 .
\end{array}\right.
$$

Proof. The case for $q=0$ is obvious. For $q>0$, this follows from the fact that the integration map defines an isomorphism $I: \operatorname{diff}(K, \mathrm{ch})^{q}(M) \stackrel{\simeq}{\longrightarrow} K^{q}(M)$ for $q>0$. Since the differential $d_{3}$ for the classical AHSS is given by $S q_{\mathbb{Z}}^{3}$ and the integration map defines an isomorphism of corresponding first quadrant spectral sequences, the case $q>0$ is settled.

For $q<0$, Corollary 15 implies that the Bockstein $\beta$ commutes with the differentials on the $E_{3}$-page. We therefore have

$$
\beta d_{3}=S q_{\mathbb{Z}}^{3} \beta=\widetilde{\beta} S q^{3} \rho_{2} \beta .
$$

Rephrasing, we have the commuting diagram

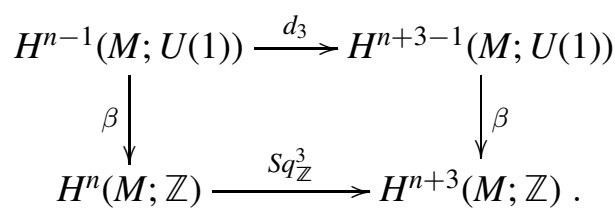

We now claim that $\tilde{\beta}=\beta \circ \Gamma_{2}$. Indeed, we have a morphism of short exact sequences

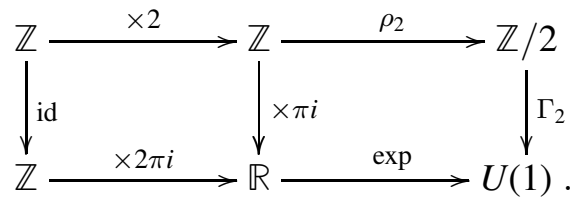

This morphism induces a morphism on the associated long exact sequences on cohomology. The homotopy commutativity of the resulting diagram, after delooping once 
to extend to the left,

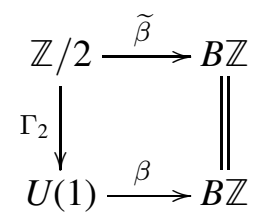

immediately establishes the claim.

Now it follows from expression (4-4) that $d_{3}-\Gamma_{2} S q^{3} \rho_{2} \beta$ is in the kernel of $\beta$. By exactness of the Bockstein, this implies that it must be in the image of the exponential map, exp : $H^{*}(-; \mathbb{R}) \rightarrow H^{*}(-; U(1))$. Hence there is an operation $\psi: H^{*}(-; U(1)) \rightarrow$ $H^{*+3}(-; \mathbb{R})$ such that

$$
\phi:=\exp \circ \psi=\exp (\psi)=d_{3}-\Gamma_{2} S q^{2} \rho_{2} \beta .
$$

Equivalently, we have a factorization

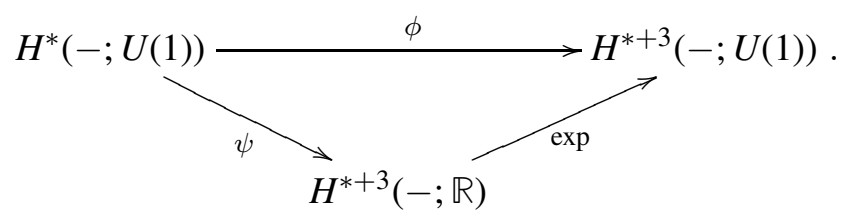

Now the group of natural transformations $H^{*}(-; U(1)) \rightarrow H^{*+3}(-; \mathbb{R})$ is in bijective correspondence with $H^{*+3}(K(U(1), *) ; \mathbb{R}) \cong 0$. Hence, $\psi=0$. 7 Consequently, $\exp \circ \psi=0$, so that $\phi=0$. Therefore, indeed we have

$$
d_{3}=\Gamma_{2} S q^{3} \rho_{2} \beta \text {. }
$$

Remark 14 The above proposition suggests that these operations are related to some sort of differential Steenrod squares. Indeed, this is the case, which has been investigated by the authors in [GS16a], with $\widehat{S q}^{3}$ being one such operation.

Now that we have established the algebraic construction, we turn to investigating the convergence of the spectral sequence from a geometric point of view. In particular, we immediately observe that the only terms in the spectral sequence which contain

\footnotetext{
${ }^{7}$ In the published version, it was erroneously claimed that $\operatorname{hom}\left(H^{n}(M ; \mathbb{R}), A\right) \cong$ $\operatorname{hom}\left(H^{n}(M ; \mathbb{Q}), A\right)$ for any abelian group. This is false, e.g. for $M=S^{n}, A=\mathbb{Q}, \operatorname{hom}(\mathbb{R}, \mathbb{Q})$ is a $\mathbb{Q}$-vector space of uncountable dimension, while $\operatorname{hom}(\mathbb{Q}, \mathbb{Q}) \cong \mathbb{Q}$. The proof has been corrected.
} 
information about differential forms are at $q=0$. These terms converge to elements in the filtered graded complex (since $q=0$ )

$$
\widehat{K}(M) / F_{1} \widehat{K}(M) \text {. }
$$

Since the filtration is given by the Čech-type filtration on $M$, we see that this quotient contains elements which have nontrivial data on all open sets, intersections, and higher intersections. For the degrees $q<0$, the filtration quotients

$$
F_{p} \widehat{K}(M) / F_{p+1} \widehat{K}(M)
$$

have trivial data below $p$-intersections.

In fact, it is not too surprising that this occurs. There is a geometric model for reduced $\widehat{K}^{0}$ which is given by the moduli stack $\coprod_{n \in \mathbb{N}} \mathbf{B} U(n)_{\text {conn }}$ of unitary bundles vector bundles, equipped with Hermetian connection. More precisely, let $\mathrm{Vect}_{\nabla}$ be the moduli stack of complex Hermetian vector bundles with Hermetian connections. It was shown in [BNV16] that, after taking the Grothendieck group completion, there is a surjection given by the cycle map

$$
\text { cycl : } \operatorname{Gr}\left(\pi_{0} \operatorname{Vect}_{\nabla}(M)\right) \rightarrow \widehat{K}^{0}(M),
$$

which, in our construction, is equivalent to

$$
\text { cycl : } \operatorname{Gr}\left(\pi_{0} \operatorname{Map}\left(M, \coprod_{n \in \mathbb{N}} \mathbf{B} U(n)_{\text {conn }}\right)\right) \rightarrow \widehat{K}^{0}(M) .
$$

Now the stack $\mathbf{B} U(n)_{\text {conn }}$ can be identified with the moduli stack obtained by taking the nerve of the action groupoid $C^{\infty}(-, U(n)) / / \Omega^{1}(-; \mathfrak{u}(n))$, with the action given by gauge transformations, where $\mathfrak{u}$ is the Lie algebra of the unitary group. Let $\left\{U_{\alpha}\right\}$ be a good open cover of $M$. Then a map $M \rightarrow \coprod_{n \in \mathbb{N}} \mathbf{B} U(n)_{\text {conn }}$ is given by the following data:

- A choice of smooth $U(n)$-valued function $g_{\alpha \beta}$ on intersections $U_{\alpha} \cap U_{\beta}$.

- A choice of local connection 1-form $\mathcal{A}_{\alpha \beta}$ on open sets $U_{\alpha}$.

This is precisely the data needed to define a unitary vector bundle on $M$.

Remark 15 More relevant to our needs though, is the fact that the effects of the filtration become transparent when taking the completion of $\coprod_{n \in \mathbb{N}} \mathbf{B} U(n)_{\text {conn }}$ as a model for $\widehat{K}^{0}$. We now see that the $q=0$ terms converge to terms which involve the data of the connection, while the $q<0$ terms contain data about bundles with trivializable connections (in particular, flat connections). 
Differential $K^{1}$-theory. We now consider odd differential K-theory $K^{1}$. In this case the representing spectrum is the unitary group $U$ itself. Viewing this as a classifying space we can write $U=B \Omega U$. Of course we are interested in the corresponding stacks. Unfortunately, we do not have the analogue of the above group-loop group relation in stacks, i.e. $U_{\text {conn }} \nsucceq \mathbf{B} \Omega U_{\text {conn }}$. Nevertheless, the machinery that we set up will work equally well for differential $K^{1}$-theory, as far as the third differential goes, i.e. $d_{3}=\widehat{S q}^{3}$ still. However, the even differential are now transgressed in degree by one, so that they are also of odd degree. This is expected as the Chern character in this case is a map to cohomology of odd degree.

The story for $\widehat{K}^{1}$ can be worked out similarly, as we indicated above. Let us expand on this in more details. In the odd case, the differential cohomology diamond takes the form

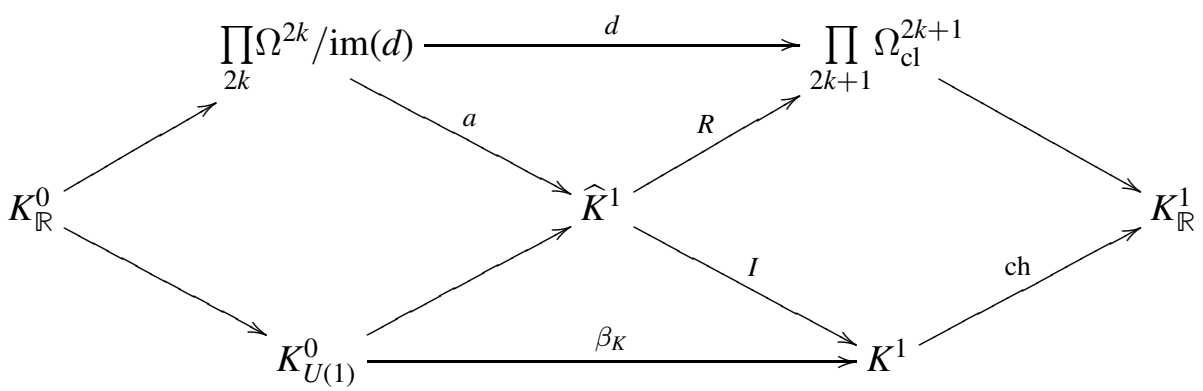

and we get a short exact sequence of presheaves (on the site of Cartesian spaces)

$$
0 \rightarrow \underline{\mathbb{Z}} \rightarrow \prod_{2 k} \Omega^{2 k} / \operatorname{im}(d) \rightarrow \widehat{K}^{1} \rightarrow 0 .
$$

It is straightforward to show that the map $\underline{\mathbb{Z}} \rightarrow \prod_{2 k} \Omega^{2 k} / \mathrm{im}(d)$ is zero. Consequently, we have the isomorphism

$$
\widehat{K}^{1} \simeq \prod_{2 k} \Omega^{2 k} / \operatorname{im}(d) \simeq \prod_{2 k+1} \Omega_{\mathrm{cl}}^{2 k+1} .
$$

Using the same type of argument as in the even K-theory $K^{0}$, we likewise get a refinement of the differential of the underlying topological theory. More precisely, we see that the first nonzero differentials appear on the $E_{3}$-page is 


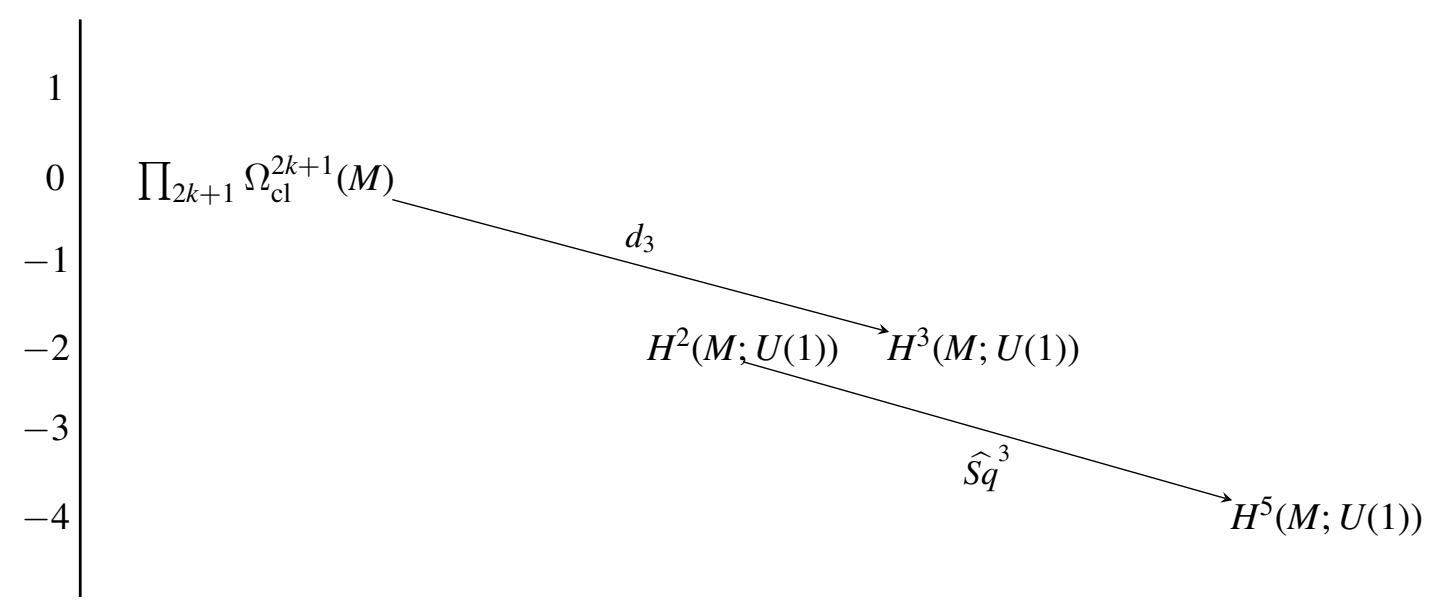

Proposition 27 Proposition 26 holds for differential $K^{1}$-theory. That is, the degree three differential in $\widehat{K}^{1}$ is given by the refinement of the Steenrod square of dimension three.

Furthermore, using the same argument as in the proof of Proposition 25, we see that the permanent cycles in bidegree $(0,0)$ are a subgroup of odd degree forms with rational periods.

Proposition 28 The group of permanent cycles in bidegree $(0,0)$ in the AHSS for $\operatorname{diff}(\Sigma K, \mathrm{ch})$ is a subgroup of odd degree closed forms with rational periods. That is, we have

$$
E_{\infty}^{0,0} \subset \prod_{k} \Omega_{\mathrm{cl}, \mathbb{Q}}^{2 k-1}(M) \oplus \mathbb{Z}
$$

Example 5 (Fields in string theory and M-theory) In the string theory and M-theory literature one encounters settings where cohomology classes are compared to $K$-theory elements, in the sense of asking when a cohomology class arises from or 'lift to' a $K$ theory class. This involves, in a sense, a physical modelling of the process of building the AHSS. One such obstruction is $S q^{3}$, viewed as the first nontrivial differential $d_{3}$ in $K$-theory, so that the condition $S q^{3} x=0$ on a cohomology class $x$ amounts to saying that the class lift to K-theory. This is desirable in the study of the partition function of the fields in type IIA string theory (see [DMW03] [KS03]). On the other hand, it is 
desirable to have differential refinements for physical purposes. Therefore, now that we have the differential AHSS at our disposal, it is natural to consider expressions such as $d_{3}(\widehat{x}):=\widehat{S q}^{3} \hat{x}=0$ on the differential cohomology class $\widehat{x}$ that refines the topological class $x$. This can be viewed as a condition on cohomology with $U(1)$-coefficients (or flat $n$-bundles), in order that they lift to flat elements in $\widehat{K} .{ }^{8}$ If the degree of the class $x$ is even then we are in type IIA string theory and we lift to differential $K^{0}$-theory. On the other hand, being in type IIB string theory means the degree of $x$ is odd, and we are lifting to differential $K^{1}$-theory. The new differentials $d_{2 m}$ and $d_{2 m+1}$ arising from differential forms will correspond to even and odd degree closed differential forms, as the particular forms representing the physical fields $F_{2 m}$ and $F_{2 m+1}$ via the Chern character.

Example 6 (D-brane charges) The charges of D-branes can a priori be taken to be given as a class in cohomology $\mathcal{Q}_{H} \in H^{*}(X ; \mathbb{Q})$. Quantum effects requires some of these charges to be (up to shifts) to be in integral cohomology. However, in order to not discuss isomorphism classes of such physical objects but pinning down a particular physical object, one considers the charges to take values in differential cohomology, with Deligne cohomology being one such presentation, $\mathcal{Q}_{\hat{H}} \in \widehat{H}^{*}(X ; \mathbb{Z})$ (see [CJM04]). On the other hand, careful analysis reveals that the charges take values in $K$-theory rather than in cohomology $\mathcal{Q}_{K} \in K^{i}(X)$, for $i=0,1$ for type IIB/IIA (see [MM97] [FW99] [BMRS08]). Such a class exists if the cohomology charge satisfies $S q^{3} \mathcal{Q}_{H}=0$. Again at this stage adding in the geometry requires the charges to take values in differential $K$-theory $\mathcal{Q}_{\widehat{K}} \in \widehat{K}^{i}(X)$. Our construction now allows for a characterization of when charges in Deligne cohomology lift to charges in differential $K$-theory,namely when they are annihilated by the third differential in the smooth AHSS, i.e. when $\widehat{S q}^{3} \mathcal{Q}_{\hat{H}}=0$.

\subsection{Differential Morava K-theory}

There are various interesting generalized cohomology theories that descend from complex cobordism, among which are Morava K-theory and Morava E-theory. Such theories can be defined using their coefficient rings, which in general are polynomials over finite or $p$-adic fields on generators whose dimension depends on the chromatic level and the prime $p$. As such, these kind of theories do not lend themselves directly

\footnotetext{
${ }^{8}$ This could end up being stronger in the sense that it is a condition for lifting differential cohomology classes to differential K-theory, but we will leave that for future investigations.
} 
to immediate geometric interpretation, in contrast to the case of K-theory, which can be formulated via stable isomorphism classes of vector bundles.

However, recent work in [LSW16] (generalizing some aspects of [BDR04]) seems to give hope in that direction. Nevertheless, just because an entity is defined over a finite field does not automatically make it ineligible for differential refinement. In fact, recently [GS16a] we have demonstrated this for the case of Steenrod cohomology operations, which are a priori $\mathbb{Z} / p$-valued operations. The main point there was that as long as these admit integral lifts then they do have a chance at a differential refinement. What we will seek here is something analogous: integral refinements of such generalized cohomology theories.

We will consider the integral Morava K-theory $\widetilde{K}(n)$, highlighted in [KS03] [Sa10] [SW15]. Morava K-theory $K(n)$ is the $\bmod p$ reduction of an integral (or $p$-adic) lift $\widetilde{K}(n)$ with coefficient ring $\widetilde{K}(n)_{*}=\mathbb{Z}_{p}\left[v_{n}, v_{n}^{-1}\right]$. This theory more closely resembles complex K-theory than is the case for the $\bmod p$ versions (for $n=1$, it is the $p$ completion of K-theory). The integral theory is much more suited to applications in physics [KS03] [Sa10] [Buh11] [SW15].

The Atiyah-Hirzebruch spectral sequence for Morava K-theory has been studied by Yagita in [Ya80] (see also [KS03]). There is a spectral sequence converging to $K(n)^{*}(X)$ with $E_{2}$-term $E_{2}^{p, q}=H^{p}\left(X, K(n)^{q}\right)$. While this can be done for any prime, we will focus on the prime 2 . In this case, the first possibly nontrivial differential is $d_{2^{n+1}-1}$; this is given by [Ya80]

$$
d_{2^{n+1}-1}\left(x v_{n}^{k}\right)=Q_{n}(x) v_{n}^{k-1} .
$$

Here $Q_{n}$ is the $n$th Milnor primitive at the prime 2, which may be defined inductively as $Q_{0}=S q^{1}$, the Bockstein operation, and $Q_{j+1}=S q^{2^{j}} Q_{j}-Q_{j} S q^{2^{j}}$, where $S q^{j}$ : $H^{n}\left(X ; \mathbb{Z}_{2}\right) \rightarrow H^{n+j}\left(X ; \mathbb{Z}_{2}\right)$ is the $j$-th Steenrod square. These operations are derivations

$$
Q_{j}(x y)=Q_{j}(x) y+(-1)^{|x|} x Q_{j}(y) .
$$

The signs are of course irrelevant at $p=2$, but will become important in the integral version. Extensive discussion of the mod $p$ Steenrod algebra in terms of these operations is given in [Ta99].

The integral theory is also computable via an AHSS, which can be deduced from [KS03] [SW15]. There is an AHSS converging to $\widetilde{K}(n)^{*}(X)$ with $E_{2}^{p, q}=H^{p}\left(X, \widetilde{K}(n)^{q}\right)$. The first possibly nontrivial differential is $d_{2^{n+1}-1}$; this is given by

$$
d_{2^{n+1}-1}\left(x v_{n}^{k}\right)=\widetilde{Q}_{n}(x) v_{n}^{k-1} .
$$

Here $\widetilde{Q}_{k}: H^{*}(X ; \mathbb{Z}) \rightarrow H^{*+2^{k+1}-1}(X ; \mathbb{Z})$ is an integral cohomology operation lifting 
the Milnor primitive $Q_{k}$.

In order to consider differential refinement of Morava K-theory, we need geometric information encoded in differential forms, hence rational information. The rationalization of Morava K-theory $\widetilde{K}(n)$, like any reasonable spectrum exists and can be thought of as localization at $\widetilde{K}(0)=H \mathbb{Q}$. See [Bo79] [Ra84]. We can in the same way localize at $\mathbb{R}$. More precisely, the localized theory is given by

$$
\widetilde{K}_{\mathbb{R}}(n)=\widetilde{K}(n) \wedge M \mathbb{R},
$$

where $M \mathbb{R}$ is an Eilenberg-Moore spectrum. We have an equivalence

$$
\widetilde{K}_{\mathbb{R}}(n) \simeq H\left(\mathbb{Z}\left[v_{n}, v_{n}^{-1}\right] \otimes \mathbb{R}\right)
$$

and a Chern character map

$$
\text { ch }: \widetilde{K}(n) \rightarrow H\left(\mathbb{Z}\left[v_{n}, v_{n}^{-1}\right] \otimes \Omega^{*}\right) .
$$

Thus we can form the differential function spectrum $\operatorname{diff}(\widetilde{K}(n), \mathrm{ch})$ and we can form the associated AHSS. To see what form the spectral sequence takes, we need to discuss the flat Morava K-theory $\widetilde{K}_{U(1)}(n)$, defined by the fiber sequence

$$
\widetilde{K}(n) \rightarrow \widetilde{K}(n) \wedge M \mathbb{R} \rightarrow \widetilde{K}_{U(1)}(n):=\widetilde{K}(n) \wedge M U(1) .
$$

This theory is periodic with period $2\left(2^{n}-1\right)$. Indeed, both $\widetilde{K}(n)$ and its rationalization are periodic and we have a long exact sequence

$$
\ldots \widetilde{K}(n)^{m}(M) \rightarrow(\widetilde{K}(n) \wedge M \mathbb{R})^{m}(M) \rightarrow \widetilde{K}_{U(1)}^{m}(n)(M) \rightarrow \widetilde{K}(n)^{m+1}(M) \rightarrow \ldots
$$

relating the flat theory to both the rational ind integral theory. This, in particular, gives the following identification.

Lemma 29 The coefficients of flat Morava K-theory are given by

$$
\widetilde{K}_{U(1)}(n)^{m}(*) \simeq\left\{\begin{array}{cl}
U(1), & m=2\left(2^{n}-1\right) \\
0, & \text { otherwise }
\end{array}\right.
$$

Knowing the coefficients of the flat theory, we can write down the relevant nonzero 
terms on the $E_{2\left(2^{n}-1\right)}$-page of the corresponding spectral sequence

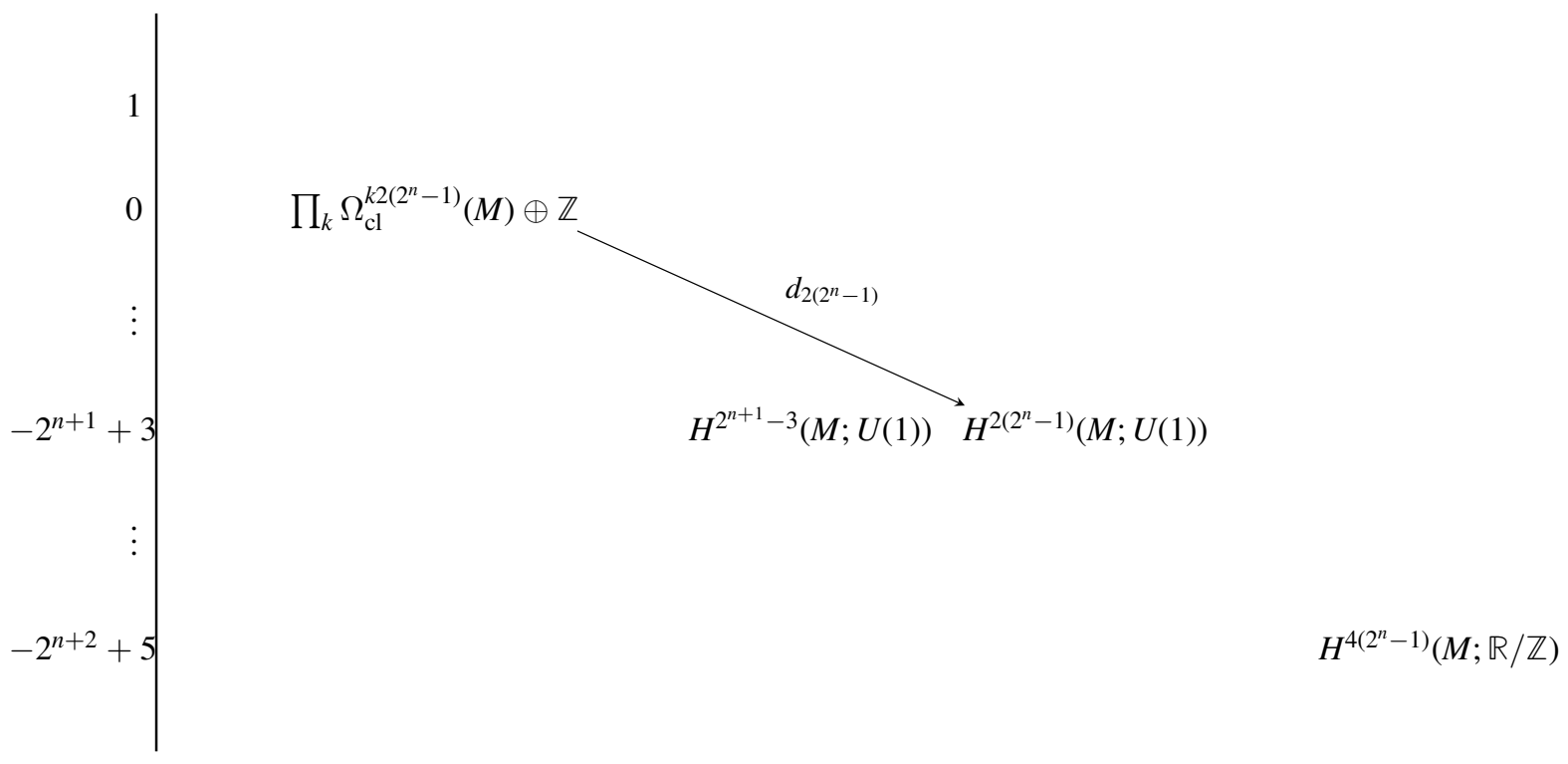

and the only nonzero differential is given by

$$
d_{2\left(2^{n}-1\right)}: \prod_{k} \Omega_{\mathrm{cl}}^{k 2\left(2^{n}-1\right)}(M) \oplus \mathbb{Z} \rightarrow H^{2\left(2^{n}-1\right)}(M ; \mathbb{R} / \mathbb{Z}) .
$$

Just as in the case for differential $K$-theory (see Propositions 25 and 28), we have the following.

Proposition 30 The group of permanent cycles in bidegree $(0,0)$ in the AHSS for $\operatorname{diff}(\widetilde{K}(n), \mathrm{ch})$ is a subgroup of certain closed forms with rational periods. More precisely, we have

$$
E_{\infty}^{0,0} \subset \prod_{k} \Omega_{\mathrm{cl}, \mathbb{Q}}^{2 k\left(2^{n}-1\right)}(M) \oplus \mathbb{Z}
$$

To identify the the Čech cohomology groups with coefficients in $\widehat{K}(n)^{0}$, we make the identification (as we did for differential $K$-theory)

$$
\widehat{K}(n)^{0} \simeq \prod_{k} \Omega_{\mathrm{cl}}^{2 k\left(2^{n}-1\right)} \oplus \underline{\mathbb{Z}}
$$

on the site of Cartesian spaces. Again, using the sheaf condition over smooth manifolds, 
we have

$$
H^{p}\left(M ; \widehat{K}(n)^{0}\right) \simeq \prod_{k} \Omega_{\mathrm{cl}}^{2 k\left(2^{n}-1\right)}(M) \oplus \mathbb{Z} .
$$

We now consider the differential refinement of the (integrally-lifted) Milnor primitive. As before, let $\Gamma_{2}: H^{n}(-; \mathbb{Z} / 2) \rightarrow H^{n}(-; U(1))$ denote the map induced by the representation of $\mathbb{Z} / 2$ as the square roots of unity and let $\rho_{2}: \mathbb{Z} \rightarrow \mathbb{Z} / 2$ denote the $\bmod 2$ reduction.

Lemma 31 The integral Milnor primitive $\widetilde{Q}_{n}$ factors through the representation $\Gamma_{2}$ : $\mathbb{Z} / 2 \hookrightarrow U(1)$. That is, there exists an operation $\widehat{Q}_{n}$ such that

$$
Q_{n} \rho_{2}=\rho_{2} \widetilde{Q}_{n}=\rho_{2} \beta \Gamma_{2} \widehat{Q}_{n},
$$

where $\beta$ is the Bockstein for the exponential sequence.

Proof. Recall first that $\rho_{2} \beta \Gamma_{2}=\rho_{2} \tilde{\beta}=S q^{1}$, where $\tilde{\beta}$ is the Bockstein for the mod 2 reduction sequence. We can therefore rewrite the above equation as

$$
Q_{n} \rho_{2}=\rho_{2} \widetilde{Q}_{n}=\rho_{2} \beta \Gamma_{2} \widehat{Q}_{n}=S q^{1} \widehat{Q}_{n} .
$$

and the existence of the class $\widehat{Q}_{n}$ holds if and only if $S q^{1} Q_{n} \rho_{2}=0$. On the other hand, the existence of the integral lift $\widetilde{Q}_{n}$ immediately implies this condition.

Again, let $\beta$ and $\tilde{\beta}$ denote the Bockstein homomorphism corresponding to the sequences $0 \rightarrow \mathbb{Z} \rightarrow \mathbb{R} \rightarrow \mathbb{R} / \mathbb{Z} \rightarrow 0$ and $0 \rightarrow \mathbb{Z} \rightarrow \mathbb{Z} \rightarrow \mathbb{Z} / 2 \rightarrow 0$, respectively. Then the following can be proved in a similar way as we did for Proposition 26 in the case of differential K-theory.

Proposition 32 (Odd differentials for Morava AHSS) The $\left(2^{n+1}-1\right)$-differential in the AHSS for differential Morava K-theory is given by

$$
d_{2^{n+1}-1}=\left\{\begin{array}{cc}
\Gamma_{2} \widehat{Q}_{n} \rho_{2} \beta, & q<0, \\
\widetilde{Q}_{n}, & q>0, \\
0, & q=0 .
\end{array}\right.
$$

Remark 16 (Odd primes) The above discussion has been for the prime 2, that is, we are considering integral Morava $K$-theory as arising from lifting of the $p=2$ Morava $K$-theory. We can do the same for odd primes, leading to integral Morava K-theory 
lifted from an odd prime $p$. A similar discussion follows and we have an integral lift of the Milnor primitive at odd primes, as in Lemma 31. The differentials will be again given by these refinement of the Milnor primitive, i.e. Proposition 32 holds except that the primitives are defined using the Steenrod reduced power operations $P^{j}$. Precisely, $Q_{0}$ is the Bockstein homomorphism associated to reduction $\bmod p$ sequence, and inductively $Q_{i+1}=P^{p^{i}} Q_{i}-Q_{i} P^{p^{i}}$. The operations $P^{j}$ have been differentially refined in [GS16a]. Hence the refinement of the Milnor primitives at odd primes will also follow. Then the $\left(p^{n+1}-1\right)$-differential in the AHSS for differential Morava K-theory is given by

$$
d_{p^{n+1}-1}=\left\{\begin{array}{cc}
\Gamma_{p} \widehat{Q}_{n} \rho_{p} \beta, & q<0, \\
\widetilde{Q}_{n}, & q>0, \\
0, & q=0 .
\end{array}\right.
$$

Example 7 (Lifting fields to differential Morava K-theory) We will build on Example 5 and aim to lift the cohomology classes beyond K-theory. In particular, for $x=\lambda=$ $\frac{1}{2} p_{1}$ the first Spin characteristic class, we have $\hat{x}=\hat{\lambda}$ the differential refinements of $\lambda$ [SSS12] [FSSt12] (which can be viewed as a lifted Wu class [HS05]) we would have $\widehat{S q}^{3} \hat{\lambda}=0$. This condition in differential cohomology can be viewed as a refinement of the condition $W_{7}=S q^{3} \lambda=0$ leading to orientation with respect to integral Morava $K(2)$-theory (lifted from the prime $p=2$ ) as shown in [KS03] and elaborated further in [Buh11]. From the structure of the smooth AHSS in relation to the classical AHSS, one can extend various results to the differential case. For instance, one can generalize the statement in [KSO3] on orientation to state that: an oriented smooth 10-dimensional manifold is oriented with respect to differential (integrally lifted from $p=2$ ) Morava $\mathrm{K}(2)$-theory $\widehat{K}(2)$ if the class $\hat{W}_{7}:=\widehat{S q}^{3} \hat{\lambda}=0$. The development of this as well as the relation to refinements of characteristic classes deserves a separate treatment and will be addressed elsewhere.

Remark 17 (i) Note that our construction allows for an AHSS for other spectra beyond the particular ones we discussed above. This holds for any spectrum which admits a rationalization, whose coefficients are known, and which can be lifted integrally in the sense that we discussed at the beginning of this section.

(ii) All the cohomology theories that we used in this paper can be twisted. Indeed, the construction in this paper can be generalized to construct an AHSS for twisted differential spectra [GS16b], in the sense of [BN14]. 


\section{Acknowledgement}

The authors would like to thank Ulrich Bunke, Thomas Nikolaus, and Craig Westerland for interesting discussions at the early stages of this project and Urs Schreiber for very useful comments on the first draft. We are grateful to the anonymous referee for a careful reading and for useful suggestions.

\section{References}

[Ad74] J. F. Adams, Stable homotopy and generalised cohomology, The Univ. of Chicago Press, Chicago, 1974.

[AH62a] M. F. Atiyah and F. Hirzebruch, Vector bundles and homogeneous spaces, 1961 Proc. Sympos. Pure Math. vol. III, pp. 7-38, American Math. Soc., Providence, R.I., 1962.

[AH62b] M. F. Atiyah and F. Hirzebruch, Analytic cycles on complex manifolds, Topology 1 (1962), 25-45.

[Ar92] D. Arlettaz, The order of the differentials in the Atiyah-Hirzebruch spectral sequence, $K$-Theory 6 (1992), no. 4, 347-361.

[BDR04] N. A. Baas, B. I. Dundas, and J. Rognes, Two-vector bundles and forms of elliptic cohomology, Topology, geometry and quantum field theory, London Math. Soc. Lecture Note Ser., vol. 308, Cambridge Univ. Press, Cambridge, 2004, pp. 18-45.

[Bj95] A. Björner, Topological Methods, Handbook of Combinatorics, North-Holland, Amsterdam, 1995, pp. 1819-1872.

[Bo79] A. K. Bousfield, The localization of spectra with respect to homology, Topology 18 (1979), no. 4, 257-281.

[BT82] R. Bott and L. W. Tu, Differential forms in algebraic topology, Springer-Verlag, New York-Berlin, 1982.

[BMRS08] J. Brodzki, V. Mathai, J. Rosenberg, and R. J. Szabo, D-Branes, RR-fields and duality on noncommutative manifolds, Commun. Math. Phys. 277 (2008), 643-706, [arXiv:hep-th/0607020].

[Br73] K. Brown, Abstract homotopy theory and generalized sheaf cohomology, Trans. Amer. Math. Soc. 186 (1973), 419-458 .

[Br93] J-L Brylinski, Loop spaces, characteristic classes and geometric quantization, Progress in Math. 107, Birkhäuser, Boston, 1993.

[BM96] J-L Brylinski and D. A. McLaughlin, Čech cocycles for characteristic classes, Communications Math. Phys. 178 (1996), 225-236.

[Buh11] L. Buhné, Properties of integral Morava K-theory and the asserted application to the Diaconescu-Moore-Witten anomaly, Diploma thesis, Hamburg University, 2011. 
[Bu12] U. Bunke, Differential cohomology, [arXiv:math. AT/1208.3961].

[BKS10] U. Bunke, M. Kreck, and T. Schick, A geometric description of differential cohomology, Ann. Math. Blaise Pascal 17 (2010), no. 1, 1-16.

[BN14] U. Bunke and T. Nikolaus, Twisted differential cohomology, [arXiv: 1406.3231].

[BNV16] U. Bunke, T. Nikolaus, and M. Völkl, Differential cohomology theories as sheaves of spectra, J. Homotopy Relat. Struct. 11 (2016), no. 1, 1-66.

[BS09] U. Bunke and T. Schick, Smooth K-theory, Astérisque 328 (2009), 45-135 (2010)

[BS10] U. Bunke and T. Schick, Uniqueness of smooth extensions of generalized cohomology theories, J. Topol. 3 (2010) 110-156.

[BSSW09] U Bunke, T Schick, I Schröder, and M Wiethaup, Landweber exact formal group laws and smooth cohomology theories, Algebr. Geom. Topol. 9 (2009) 1751-1790.

[CJM04] A. L. Carey, S. Johnson, and M. K. Murray, Holonomy on D-branes, J. Geom. Phys. 52 (2004), 186-216, [arXiv: hep-th/0204199].

[CS85] J. Cheeger and J. Simons, Differential characters and geometric invariants, volume 1167 of Lecture Notes in Math., pages 50-80. Springer, Berlin, 1985.

[DMW03] E. Diaconescu, G. Moore, and E. Witten, $E_{8}$ gauge theory, and a derivation of K-theory from M-theory, Adv. Theor. Math. Phys. 6 (2003) 1031-1134, [arXiv:hep-th/0005090].

[Do62] A. Dold, Relations between ordinary and extraordinary cohomology, Colloq. Algebr. Topology, Aarhus, 1962, 2-9.

[Du01] D. Dugger, Combinatorial model categories have presentations, Adv. Math. 164 (2001), no. 1, 177-201, [arXiv:math/0007068] [math. AT].

[DL05] J. L. Dupont and R. Ljungmann, Integration of simplicial forms and Deligne cohomology, Math. Scand. 97 (2005), no. 1, p. 11-39.

[EV92] H. Esnault and E. Viehweg, Lectures on Vanishing Theorems, Birkhäuser, DMV Seminar 20, 1992.

[FSS13] D. Fiorenza, H. Sati, and U. Schreiber, Extended higher cup-product Chern-Simons theory, J. Geom. Phys. 74 (2013), 130-163, [arXiv: 1207.5449] [hep-th].

[FSS15a] D. Fiorenza, H. Sati, and U. Schreiber, A Higher stacky perspective on ChernSimons theory, Mathematical Aspects of Quantum Field Theories (Damien Calaque and Thomas Strobl eds.), Springer, Berlin (2015), [arXiv : 1301 . 2580] [hep-th].

[FSSt12] D. Fiorenza, U. Schreiber, and J. Stasheff, Čech cocycles for differential characteristic classes - An infinity-Lie theoretic construction, Adv. Theor. Math. Phys. 16 (2012), 149-250, [arXiv: 1011.4735] [math. AT].

[FFG86] A. T. Fomenko, D. B. Fuchs, and V. L. Gutenmacher, Homotopic Topology, Akamémiai Kiadó, Budapest, 1986. 
[Fr00] D. S. Freed, Dirac charge quantization and generalized differential cohomology, Surv. Diff. Geom., VII, pages 129-194, Int. Press, Somerville, MA, 2000.

[FL10] D. S. Freed and J. Lott, An index theorem in differential K-theory, Geom. \& Top. 14 (2010), 903-966.

[FW99] D. S. Freed and E. Witten, Anomalies in string theory with D-branes, Asian J. Math. 3 (1999), 819, [arXiv:hep-th/9907189].

[Ga97] P Gajer, Geometry of Deligne cohomology, Invent. Math. 127 (1997) 155-207.

[GL15] A. Gorokhovsky and J. Lott, A Hilbert bundle description of differential K-theory, [arXiv: 1512.07185] [math.DG].

[GS15] D. Grady and H. Sati, Massey products in differential cohomology via stacks, [arXiv: 1510.06366] [math.AT].

[GS16a] D. Grady and H. Sati, Primary operations in differential cohomology, [arXiv: 1604. 05988] [math.AT].

[GS16b] D. Grady and H. Sati, AHSS for twisted differential spectra, to appear.

[GM81] P.A. Griffiths and J.W. Morgan, Rational homotopy theory and differential forms, Progress in Mathematics, 16, Birkhäuser, Boston, Mass., 1981.

[Ha02] A. Hatcher, Algebraic topology, Cambridge University Press, Cambridge, 2002.

[HMSV15] P. Hekmati, M. K. Murray, V. S. Schlegel, and R. F. Vozzo, A Geometric model for odd differential K-theory, Diff. Geom. Appl. 40 (2015), 123-158, [arXiv: 1309. 2834] [math.KT].

[Hi71] P. J. Hilton, General Cohomology and K-theory, Cambridge University Press, Cambridge, 1971.

[Ho12] M.-H. Ho, The differential analytic index in Simons-Sullivan differential K-theory, Ann. Global Anal. Geom. 42 (2012), 523-535, [arXiv: 1110.0151] [math.DG].

[Ho14] M.-H. Ho, Remarks on flat and differential K-theory, Ann. Math. Blaise Pascal 21 (2014), 91-101, [arXiv: 1203. 5383] [math.DG].

[HS05] M. J. Hopkins and I. M. Singer, Quadratic functions in geometry, topology, and M-theory, J. Differential Geom. 70 (3) (2005), 329-452.

[HJJS08] D. Husemöller, M. Joachim, B. Jurco, and M. Schottenloher, Basic Bundle Theory and K-Cohomology Invariants, Springer, Berlin, 2008.

[Ja87] J. F. Jardine, Stable homotopy theory of simplicial presheaves, Canadian J. Math. 39 (1987), 733-747.

[Ja15] J. F. Jardine, Local Homotopy Theory, Springer-Verlag, New York, 2015.

[Ka87] M. Karoubi, Homologie cyclique et K-théorie, Astérisque no. 149 (1987).

[K108] K. R. Klonoff, An index theorem in differential K-theory, PhD Thesis, The University of Texas at Austin, 2008. 
[KS03] I. Kriz and H. Sati, M-theory, type IIA superstrings, and elliptic cohomology, Adv. Theor. Math. Phys. 8 (2004) 345, [arXiv : hep-th/0404013].

[LSW16] J. A. Lind, H. Sati, and C. Westerland, A higher categorical analogue of topological T-duality for sphere bundles, [arXiv: 1601.06285] [math.AT].

[Lo94] J. Lott, $\mathbb{R} / \mathbb{Z}$ index theory, Comm. Anal. Geom. 2 (1994), no. 2, 279-311.

[Lo00] J. Lott, Secondary analytic indices, Progr. Math., 171, 231-293, Birkhäuser Boston, Boston, MA, 2000.

[Lu10] J. Lurie, Course notes on Chromatic Homotopy Theory, lecture 25 (2010), http://www. math.harvard.edu/ lurie/252x.html

[Lu11] J. Lurie, Higher algebra, prepublication book draft, available at http://www.math.harvard.edu/ lurie/, 2011.

[Ma63] C. R. F. Maunder, The spectral sequence of an extraordinary cohomology theory, Proc. Cambridge Philos. Soc. 59 (1963), 567-574.

[MS06] J. P. May and J. Sigurdsson, Parametrized Homotopy Theory, Amer. Math. Soc., Providence, RI, 2006.

[Mc01] J. McCleary, A User's Guide to Spectral Sequences, 2nd ed., Cambridge University Press, Cambridge, 2001.

[MM97] R. Minasian and G. Moore, $K$-theory and Ramond-Ramond charge, J. High Energy Phys. 9711 (1997), 002, [arXiv: hep-th/9710230].

[Mi81] H. R. Miller, On relations between Adams spectral sequences, with an application to the stable homotopy of a Moore space, J. Pure Appl. Algebra 20 (1981), no. 3, 287-312.

[Pr06] V. V. Prasolov, Elements of Combinatorial and Differential Topology, Amer. Math. Soc., Providence, RI, 2006.

[Ra84] D. C. Ravenel, Localization with respect to certain periodic homology theories, Amer. J. Math. 106 (1984), no. 2, 351-414.

[Ru08] Y. Rudyak, On Thom Spectra, Orientability and Cobordism, Springer-Verlag, Berlin, 2008.

[Sa10] H. Sati, Geometric and topological structures related to M-branes, In Superstrings, geometry, topology, and $C^{*}$-algebras, 181-236, Proc. Sympos. Pure Math., 81, Amer. Math. Soc., Providence, RI, 2010, [arXiv : 1001 .5020] [math.DG].

[SSS12] H. Sati, U. Schreiber, and J. Stasheff, Differential twisted String- and Fivebrane structures, Commun. Math. Phys. 315 (2012), 169-213, [arXiv : 0910 . 4001] [math . AT].

[SW15] H. Sati and C. Westerland, Twisted Morava K-theory and E-theory, J. Topol. 8 (2015), no. 4, 887-916, [arXiv:1109.3867] [math.AT].

[Sc13] U. Schreiber, Differential cohomology in a cohesive infinity-topos, [arXiv: 1310.7930] [math-ph].

[Sch] S. Schwede, Symmetric Spectra, book draft http://www.math.uni-bonn.de/people/schwede/SymSpec-v3.pdf 
[Sh07] B. Shipley. HZZZ-algebra spectra are differential graded algebras, Amer. J. Math. 129 (2) (2007), 351-379.

[SS08] J. Simons and D. Sullivan, Axiomatic characterization of ordinary differential cohomology J. Topol. 1(1) (2008), 45-56.

[Sw75] R. Switzer, Algebraic Topology, Springer-Verlag, Berlin, 1975.

[Ta99] H. Tamanoi, $\mathcal{L}$-subalgebras, Milnor basis, and cohomology of Eilenberg-MacLane spaces, J. Pure Applied Alg. 137 (1999), 153-198.

[TWZ13] T. Tradler, S. O. Wilson, and M. Zeinalian, An elementary differential extension of odd K-theory, J. K-Theory 12 (2013), no. 2, 331-361.

[TWZ15] T. Tradler, S. O. Wilson, and M. Zeinalian, Differential K-theory as equivalence classes of maps to Grassmannians and unitary groups, [arXiv:1507.01770].

[Up14] M. Upmeier, Refinements of the Chern-Dold character: Cocycle additions in differential cohomology, [arXiv: 1404 . 2027] [math.AT].

[Up15] M. Upmeier, Algebraic structure and integration maps in cocycle models for differential cohomology, Algebr. Geom. Topol. 15 (2015), no. 1, 65-83.

[Ya80] N. Yagita, On the Steenrod algebra of Morava K-theory, J. London Math. Soc. 22 (3) (1980), 423-438.

Department of Mathematics, New York University

Abu Dhabi, Abu Dhabi, UAE

Department of Mathematics, New York University

Abu Dhabi, Abu Dhabi, UAE

Department of Mathematics, University of Pittsburgh

Pittsburgh, PA 15260, USA

djg9@nyu.edu, hsati@nyu.edu 